\title{
"Friends Are Thieves of Time": Heuristic Attention Sharing in Stable Friendship Networks
}

Citation for published version (APA):

Tenev, A. P. (2020). "Friends Are Thieves of Time": Heuristic Attention Sharing in Stable Friendship Networks. Maastricht University, Graduate School of Business and Economics. GSBE Research Memoranda No. 026 https://doi.org/10.26481/umagsb.2020026

Document status and date:

Published: 12/10/2020

DOI:

10.26481/umagsb.2020026

Document Version:

Publisher's PDF, also known as Version of record

\section{Please check the document version of this publication:}

- A submitted manuscript is the version of the article upon submission and before peer-review. There can be important differences between the submitted version and the official published version of record.

People interested in the research are advised to contact the author for the final version of the publication, or visit the DOI to the publisher's website.

- The final author version and the galley proof are versions of the publication after peer review.

- The final published version features the final layout of the paper including the volume, issue and page numbers.

Link to publication

\footnotetext{
General rights rights.

- You may freely distribute the URL identifying the publication in the public portal. please follow below link for the End User Agreement:

www.umlib.nl/taverne-license

Take down policy

If you believe that this document breaches copyright please contact us at:

repository@maastrichtuniversity.nl

providing details and we will investigate your claim.
}

Copyright and moral rights for the publications made accessible in the public portal are retained by the authors and/or other copyright owners and it is a condition of accessing publications that users recognise and abide by the legal requirements associated with these

- Users may download and print one copy of any publication from the public portal for the purpose of private study or research.

- You may not further distribute the material or use it for any profit-making activity or commercial gain

If the publication is distributed under the terms of Article $25 \mathrm{fa}$ of the Dutch Copyright Act, indicated by the "Taverne" license above, 
Anastas P. Tenev

"Friends Are Thieves of Time": Heuristic Attention Sharing in Stable Friendship Networks

RM/20/026

ISSN: $2666-8807$

\section{GSBE}

Maastricht University School of Business and Economics

Graduate School of Business and Economics

P.O Box 616

NL-6200 MD Maastricht

The Netherlands 


\title{
"Friends Are Thieves of Time": Heuristic Attention Sharing in Stable Friendship Networks
}

\author{
Anastas P. Teneva
}

\begin{abstract}
This paper studies a model of network formation in which agents create links following a simple heuristic - they invest their limited resources proportionally more in neighbours who have fewer links. This decision rule captures the notion that when considering social value more connected agents are on average less beneficial as neighbours and node degree is a useful proxy when payoffs are difficult to compute. The decision rule illustrates an externalities effect whereby an agent's actions also influence his neighbours' neighbours. Besides complete networks and fragmented networks with complete components, the pairwise stable networks produced by this model include many non-standard ones with characteristics observed in real life networks like clustering and irregular components. Multiple stable states can develop from the same initial structure - the stable networks could have cliques linked by intermediary agents while sometimes they have a core-periphery structure. The observed pairwise stable networks have close to optimal welfare. This limited loss of welfare is due to the fact that when a link is established, this is beneficial to the linking agents, but makes them less attractive as neighbours for others, thereby partially internalising the externalities the new connection has generated.
\end{abstract}

Keywords: Networks; Heuristics; Bilateral communication links; Social value JEL Classification: A13, C72, D85

\section{Introduction and Motivation}

This paper studies the decentrally emerging stable networks when all agents employ a simple decision rule and invest proportionally more in neighbours who have a lower degree. In the

\footnotetext{
${ }^{a}$ Department of Microeconomics and Public Economics, Maastricht University, P.O. Box 616, 6200 MD Maastricht, The Netherlands (e-mail: ap.tenev@maastrichtuniversity.nl). This project has benefited greatly from the useful feedback at: seminar talks in Maastricht University, the Netherlands and the Otago Business School in Dunedin, New Zealand; poster presentation at the Conference in Honour of Hans Peters in Maastricht; conference talks at SING15 in Turku, Finland and at the International Conference on Game Theory in Stony Brook, USA. The author is grateful to Jean-Jacques Herings, Dominik Karos, Ronald Peeters and Frank Thuijsman for their invaluable help and comments. The quote in the title is attributed to Francis Bacon.
} 
absence of complex optimisation at the level of every network link (cf. Brueckner (2006), Deroian (2009), Bloch and Dutta (2009), Salonen (2016), So (2016), Bourlès et al. (2017), Baumann (forthcoming)) as opposed to the node/agent level, a common assumption in the literature has been that agents divide their limited resources equally amongst their neighbours. The Jackson and Wolinsky (1996) co-author model is a prime example of such a setup. ${ }^{1}$ While a proportional allocation of resources, e.g. time, is a fair assumption in such a context, other social interactions could be driven by a different dynamic. Take a friendship network, for example. If one is interested in developing an enduring and deep friendship, this requires big investment from both parties and people do not usually allocate their time and effort equally amongst everyone they know - they have friends they see every day but they also have acquaintances with whom they have just minute-long conversations. Since no one wants to be on the more giving end of a "one-sided" friendship where the attention given is much more than the attention one gets, a rational friend-seeker would try to give more time/attention to people who are expected to return it. Even within the co-author context it would be wise to expect a smaller contribution from a co-author who is involved in many projects. In the presence of synergies and assuming that time and effort are the most important factors determining the quality of a paper one would be better off investing more in co-authors who are involved in fewer projects and less in co-authors who are involved in more projects to have higher utility, ceteris paribus. In the extreme cases, if author A connects with author B who has only one project, the common project $\mathrm{AB}$ would be finished much faster compared to a situation in which $\mathrm{A}$ connects to author $\mathrm{C}$ who has ten projects. If author $\mathrm{C}$ wants to allocate any time to even one of the remaining nine projects, he would have to spend strictly less than all of his research time on the common project AC.

Harmsen-van Hout et al. (2016) show experimentally that when faced with linking choices involving increased complexity of payoffs, experimental subjects resort to simplified decision rules, i.e. heuristics. ${ }^{2}$ The authors identify two factors that are related to payoffs only qualitatively, but have been used by the experimental subjects: whether or not the choice option involves a deviation from the status quo and the number of direct neighbours of the (potential) linking partner. They call for future models to include the human tendencies ${ }^{3}$ "to base complex linking decisions on heuristic cues like... node degree rather than exact payoff" (Harmsen-van Hout et al. 2016), as found by their analysis.

Addressing these findings, the current paper investigates what happens if agents decide to form stronger connections with neighbours who have fewer links. More precisely, the setup

\footnotetext{
${ }^{1}$ Consider also Albornoz et al. (forthcoming) and Harmsen-van Hout et al. (2013) which have similar assumptions. Bala and Goyal (2000) and Galeotti et al. (2010) assume that links are of intensity either 0 or 1.

${ }^{2} \mathrm{~A}$ related finding is discussed by Kováŕík et al. (2018) in the context of learning where "people facing more complex environments... seem to resort to simpler learning rules."

${ }^{3}$ Consider also Hämäläinen et al. (2013) who urge researchers to include behavioural effects in OR processes.
} 
assumes that link investments are proportionally greater when they are formed with nodes of a lower degree (since the connections are bilateral, in-degree is the same as out-degree). The heuristic in the current setup is intuitively appealing, since a self-interested agent would try to maximise his potential payoff and agents who are less connected have on average more resources to allocate to a specific link. The benefit is higher if the degree of the potential neighbour is lower, since he would have to spend his resources on fewer people. ${ }^{4}$ If the agents are unaware of the exact functional form of the utility per link or if they cannot have a complete overview of the system they are part of (i.e. they have cognitive limitations), the degree gives an indication of how much one can benefit from a specific potential neighbour (on average). Using such a "rule of thumb", the model diverges from the literature on best responses to specific investment that the agents could get from their neighbours and enters the realm of heuristics-driven decisions.

One of the main objectives of the current study is to show that using a heuristic different than the equal split but equally plausible and intuitively appealing results in a highly nontrivial change in the predictions of the model, bringing it closer to observed reality. Namely, the pairwise stable networks in the current setup can have irregular components and clusters, something characteristic of social networks (Jackson and Rogers 2007). Moreover, they have close to optimal welfare. This all is achieved at a very low cost - a decision rule that does not require complicated computations or complex strategic considerations like best responses or farsightedness, ${ }^{5}$ for instance. There are also no assumptions like homophily as all nodes are identical at the start and their potential matchings are random. ${ }^{6}$ Here, it is crucial that a distinction is made between the social and the informational value of links (Harmsen-van Hout et al. 2013). In particular, this setup assumes that social (direct) value is the source of utility as would be the case in personal relationships between friends, rather than the informational (indirect) value. In this sense, people wanting to be connected to the most popular nodes (in school, for example) want that for the indirect value (getting some of the popularity), not because of the direct impact that would have (support in difficult times, for instance).

This model bears a crucial similarity with the co-author model (Jackson and Wolinsky 1996) in that it depends on the degree of the agents. There the indirect connections, the coauthor's co-authors, influence the payoff of an agent because they take from the co-author's time and so impose an externality on the co-author's co-authors. The setup of the current

\footnotetext{
${ }^{4} \mathrm{~A}$ similar logic is also captured in the Horse race betting model where betting on each horse proportional to its probability of winning is log-optimal (Cover and Thomas 2006).

${ }^{5}$ Morbitzer et al. (2014) show through simulations that the co-author model can produce a number of irregular networks when the agents exhibit farsightedness.

${ }^{6}$ Homophily, whereby similar types of nodes are more likely to be connected than dissimilar ones, tends to characterise all social networks (McPherson et al. 2001), and produces clustering. Consider Bramoullé et al. (2012) who, building on the work of Jackson and Rogers (2007), introduce individual heterogeneity to investigate homophily as a result of biased meeting processes.
} 
paper captures a more elaborate model of indirect (positive) externalities. For example, in a situation in which $\mathrm{A}$ is connected to $\mathrm{B}$ and $\mathrm{X}$, while $\mathrm{B}$ is connected to $\mathrm{A}$ and $\mathrm{Y}$, if $\mathrm{B}$ decides to create a new connection (say, to Z), this affects not only B's contribution to A and A's investment in B. It is interesting to note that through the second effect A's investment in X will also increase. In fact, B's decision to form a new link positively influences his neighbours' neighbour, while affecting his direct neighbours negatively. In its core idea of nodes differentiating the investment they commit to their neighbours this paper is closest to Baumann (forthcoming). She analyses a model of weighted network formation and characterises the types of equilibria that occur. However, there players can invest in others or in themselves and the equilibria are neither pairwise stable nor efficient.

The remainder of the paper starts by outlining the model and considering some of its welfare properties (Section 2). It proceeds to analyse regular and commonly used network structures that can be approached analytically within the current framework (Section 3), contrasting the outcomes with the results seen when the resources are equally split amongst all neighbours. Section 4 is devoted to the analysis of the types of stable networks with irregular components that emerge from simulations of the current model. The last section concludes the discussion.

\section{Model}

There is a set of agents $N \in\{1,2, \ldots, n\}$, who form a network. A connection between two nodes can be sustained only with a positive investment from both parties. The payoff from a specific link depends positively on the bilateral investment of the involved agents. For all nodes $i$ of the network, if $t_{i j}$ is the contribution that agent $i$ has in his link with agent $j$, investments in links are constrained to $t_{i j}>0$ (if investments are 0 there is no link). Additionally, if $N_{i}$ is the set of node $i^{\prime}$ s neighbours, $\sum_{j \in N_{i}} t_{i j}=1$ for all $i$, one being the highest contribution a node can make on a single link. Nodes cannot invest in themselves and therefore they always invest all their resources (a node with no links will have zero utility). There are no additional explicit costs for maintaining links except the contribution to the link, which is an opportunity cost of not investing in others. The investment in connections with neighbours is based on the following heuristic: links are proportionally stronger when they are formed with nodes of a lower degree. This means that in an equal-degree network every node spreads its resource of 1 equally amongst its neighbours, but also that if a node has two neighbours, one with degree 2 and the other one with degree 1 , the neighbour with degree 1 will get an investment twice as big as the other neighbour. Therefore, the investment per link is inversely proportional to the degree of the node it connects to, so $t_{i k}=\frac{1 / d_{k}}{\sum_{j \in N_{i}} 1 / d_{j}}$, with $d_{i}$ being node $i$ 's degree. The payoff of a specific link is given by $\sqrt{t_{i j} t_{j i}}$, which exhibits constant returns 
to scale. ${ }^{78}$ Such a functional form ensures that spreading resources between neighbours can be more beneficial than investing in only one neighbour. Following the specified heuristic for spreading resources between links, the payoff of every node $i$ can be expressed and rearranged in the following way:

$$
u_{i}=\sum_{k \in N_{i}} \sqrt{\frac{\frac{1}{d_{k}}}{\sum_{j \in N_{i}} \frac{1}{d_{j}}} * \frac{\frac{1}{d_{i}}}{\sum_{\ell \in N_{k}} \frac{1}{d_{\ell}}}}=\frac{1}{\sqrt{\sum_{j \in N_{i}} \frac{d_{i}}{d_{j}}}}\left(\sum_{k \in N_{i}} \frac{1}{\sqrt{\sum_{\ell \in N_{k}} \frac{d_{k}}{d_{\ell}}}}\right) .
$$

In other words, according to the heuristic, the payoff of an agent is a function of the ratio of the degree of the agent compared to all his neighbours' degrees and the ratio between the degree of each one of the agent's neighbours and the neighbours' neighbours.

\section{Efficiency}

As usually, a regular network refers to a network where every node has the same degree. Additionally, in this paper the term regular components/networks will only be used for components/networks with degrees greater than or equal to one, i.e. ones which have no isolated nodes.

Lemma 1. The payoff for every node in a regular network is 1 .

Proof. Follows directly from the payoff expression and the fact that the degree of all nodes is the same, i.e. $d_{i}=d_{j}=d_{k}=d_{\ell}$ and $\left|N_{i}\right|=\left|N_{k}\right|$.

$$
\frac{1}{\sqrt{\sum_{j \in N_{i}} \frac{d_{i}}{d_{j}}}}\left(\sum_{k \in N_{i}} \frac{1}{\sqrt{\sum_{\ell \in N_{k}} \frac{d_{k}}{d_{\ell}}}}\right)=\frac{1}{\sqrt{\left|N_{i}\right|}} \frac{\left|N_{i}\right|}{\sqrt{\left|N_{k}\right|}}=1 .
$$

Proposition 1. The maximum utilitarian welfare of a network with $n$ nodes is $n$.

Proof. It follows from Lemma 1 that the welfare is $n$ when the network is complete. This means that the sum of all nodes' potential contributions $n * 1$ has been recovered after the investment in the network. In order to get welfare of more than $n$ in a stable state some nodes must get more than their overall payoff of 1 . Moreover, this has to compensate for the ones that get less than 1 (if there are such nodes). For a node to get more than 1 overall payoff, it has to get more than its investment in a link from at least one link. In other words,

\footnotetext{
${ }^{7}$ The results are qualitatively similar for other concave functions.

${ }^{8} \mathrm{~A}$ similar payoff function is used in the occupational choice model with spillovers by Albornoz et al. (forthcoming).
} 
for a link between node $i$ with contribution $t_{i j}$ and node $j$ with contribution $t_{j i}$ to provide a higher return for $i$, the following needs to hold: $t_{i j}<\sqrt{t_{i j} t_{j i}}$, or $t_{i j}<t_{j i}$. This implies that the return for $j$ would be strictly smaller. If the net gain of every link (the combined gains of both sides $i$ and $j$ compared to their investments $t_{i j}$ and $t_{j i}$ in the link) in the network is negative, then the welfare will be strictly smaller than $n$. So, for utilitarian welfare greater than or equal to $n$ at least one link should have a non-negative net gain. In other words, the sum of the two investments in the link $t_{i j}$ and $t_{j i}$ and the return to the two players $2 \sqrt{t_{i j} t_{j i}}$ should be positive:

$$
-t_{i j}-t_{j i}+2 \sqrt{t_{i j} t_{j i}} \geq 0 \Leftrightarrow\left(\sqrt{t_{i j}}-\sqrt{t_{j i}}\right)^{2} \leq 0 .
$$

This is only possible for $t_{i j}=t_{j i}$ and then all investments are recovered, so there is a payoff of 1 for every node, for a total welfare of $n$.

Corollary 1. A network consisting of regular components has maximum utilitarian welfare.

Utilitarian welfare is not the only notion of welfare, another prominent measure is Rawlsian welfare, measured by the agent who is worst-off.

Proposition 2. The maximum Rawlsian welfare of a network with $n$ nodes is 1 .

Proof. Rawlsian welfare has an upper bound that is implied by the reasoning suggested in Proposition 1. In order for it to be bigger, all nodes need to have payoff higher than 1, which would contradict Proposition 1.

The propositions above establish that in the current model the most efficient networks are the ones with regular components. Since this paper focuses on social interactions and they rarely if ever produce fully regular networks, the results on efficiency present merely a benchmark against which all stable networks can be measured.

\section{Analytical observations}

This section looks into networks frequently appearing in the literature to identify which ones are pairwise stable in the current setup. This paper uses the notion of pairwise stability (Jackson and Wolinsky 1996), whereby links do not exist if one of the parties is strictly worse off with the link (they are severed unilaterally) and both nodes need to agree to form a link (bilateral consent). More formally, in a network if $u_{i}, u_{j}$ are the utilities of nodes $i$ and $j$ for $i \in N_{j}$ and $j \in N_{i}$ and $u_{i}^{\prime}, u_{j}^{\prime}$ are the utilities of the same nodes $i$ and $j$ for $i \notin N_{j}$ and $j \notin N_{i}$, keeping all other links constant, a network is pairwise stable if it holds that: (i) $u_{i} \geq u_{i}^{\prime}$ and $u_{j} \geq u_{j}^{\prime}$, for all existing links $i j$, with at least one inequality being strict; and (ii) if $u_{i}^{\prime}<u_{i}$, then $u_{j}^{\prime}>u_{j}$ for all non-existing links $i j$. 


\section{Complete Networks}

Proposition 3. The complete network is stable.

Proof. In a fully connected graph every node has payoff 1 . In order for the complete graph to be stable, removing a link should be equally good or worse than the status quo for both nodes that are connected so that they decide not to disconnect. For a complete graph with $n$ nodes this implies that after disconnecting the two nodes have degrees $n-2$, while the other $n-2$ nodes keep their degrees of $n-1$. The two nodes with degrees $n-2$ each have $n-2$ neighbours with degrees $n-1$, while the nodes with degrees $n-1$ have two neighbours with degrees $n-2$ and $n-3$ neighbours with degrees $n-1$. This is equivalent to:

$$
\begin{aligned}
\frac{1}{\sqrt{\frac{(n-2)^{2}}{n-1}}}\left(\frac{n-2}{\sqrt{2 \frac{n-1}{n-2}+\frac{n-1}{n-1}(n-3)}}\right) & \leq 1 \Leftrightarrow \frac{\sqrt{n-1}}{n-2}\left(\frac{n-2}{\sqrt{\frac{2 n-2+(n-3)(n-2)}{n-2}}}\right) \leq 1 \Leftrightarrow \\
(n-1)(n-2) & \leq 2 n-2+(n-3)(n-2) .
\end{aligned}
$$

This always holds for $n>2$. Therefore, no two nodes would decide to disconnect in a complete graph with $n>2$. If the network has $n=2$, it is clearly stable as disconnecting would bring both nodes 0 .

This establishes existence of stable networks since for any $n$ there is a stable network that is also efficient. While the complete network is stable and it achieves maximum welfare, it might not be possible to always reach it. Fortunately, it is possible to construct paths for reaching it from the empty network as long as there is a positive probability of adding a link at any step of the process.

Proposition 4. The complete network is reachable from the empty network.

Proof. See Appendix B.1.

The following proposition condenses what is known from the previous observations about regular networks and introduces a short discussion of the networks which are not pairwise stable under the current setup.

Proposition 5. A regular network is stable if and only if it is complete.

Proof. See Appendix A.

Proposition 5 shows that some of the potentially welfare maximising networks, like networks with incomplete regular components, are not stable. This is an interesting result as high regularity in components is indeed not characteristic of social networks. ${ }^{9}$

\footnotetext{
${ }^{9} \mathrm{Cf}$. for example the discussion in Jackson and Rogers (2007) of the relatively small distance between any pair of nodes and the fact that there are more nodes with relatively high and low degrees in social networks.
} 


\section{Unstable Incomplete Networks}

Proposition 5 gives the following corollary.

Corollary 2. The circle network with $n \geq 4$ nodes is not stable.

Proposition 6 below, just like the corollary above, shows that often used and investigated network types are all unstable in the current setting.

Proposition 6. The line, star with $n \geq 3$ peripheral nodes, wheel with $n \geq 4$ peripheral nodes and biregular graphs are not stable. ${ }^{10}$

Proof. See Appendix A.

\section{Networks with Complete Components}

Besides complete networks, networks with complete components can also be pairwise stable. As observed above, such networks would also exhibit maximum welfare.

Proposition 7. A network consisting of two disconnected complete components with sizes $m_{1}, m_{2} \geq 2$ such that $m_{1}+m_{2}=n$ is stable if and only if $\left|m_{1}-m_{2}\right| \geq 2$.

Proof. See Appendix A.

Corollary 3. A network consisting of $k$ disconnected complete components of respective sizes $m_{1}, \ldots, m_{k} \geq 2$ such that $\sum_{j=1}^{k} m_{j}=n$ is stable if and only if $\left|m_{j}-m_{j^{\prime}}\right| \geq 2$ for all $j, j^{\prime}=1, \ldots, k$ with $j \neq j^{\prime}$.

To interpret the result above in the context of a friendship network, one can imagine that having a few good friends is as good as having many marginal friends in terms of social value. In this case they form a group in which everyone gets equal attention from their friends which is a qualitatively different result from Proposition 5. A graph with regular components is not sufficient for stability, the components need to be complete - getting equal treatment from your friends is only stable when you are a part of a tight social group.

The following statement shows that the observations made so far are the only ones needed to analyse the stable states for small networks with $n \leq 11$ nodes. After that the model also produces stable networks with irregular components. They are discussed in the next section. This proposition is given without an analytical proof since it has been verified by checking all possible options with a computer.

Proposition 8. In graphs with:

\footnotetext{
${ }^{10}$ Biregular graphs are bipartite graphs in which vertices in the same subset of nodes of the given bipartition have the same degree.
} 
(a) $n \leq 5$ nodes the complete network is the only stable network;

(b) $5 \leq n \leq 11$ nodes the only stable networks are the complete network and networks consisting of two disconnected complete components with sizes $m_{1}, m_{2} \geq 2$ such that $m_{1}+m_{2}=n$ and $\left|m_{1}-m_{2}\right| \geq 2$.

Proof. a) The propositions above exclude many potential networks. Others are excluded for having a node unconnected to any other nodes. These cases are not stable because the isolated node would want to connect and the others would want to connect with it. The rest can be verified by a case-by-case check. b) Verified on a case-by-case basis. ${ }^{11}$

In other words, for $n \leq 11$ Propositions 5 and 7 describe all stable networks, since there can be no more than two fully connected disjoint parts for these cases.

At this stage it is instructive to contrast the results of the current setup with the situation in which everything is the same (in particular the payoff function) except that the heuristic applied is equal split of resources amongst all neighbours, i.e. $t_{i j}=1 / d_{i}$ for all $i \in N_{i}$.

Proposition 9. Under equal split of resources the stable networks have no incomplete or irregular components. ${ }^{12}$

Proof. See Appendix A.

Stated alternatively, under equal split the stable networks can only be the complete network or networks consisting of disjoint complete components. In light of Proposition 9, the results described above mirror the ones for the case when resources are spread equally amongst the neighbours. The networks under equal split also exhibit maximum efficiency as per Corollary 1. There is, however, one major difference between the current setup and the equal split. Under equal splitting of resources there are no other stable networks but ones consisting of complete subgraphs. It is important to note that only changing the way resources are split between a node's neighbours from equal to the current heuristic produces a much bigger and diverse set of stable networks. ${ }^{13}$ They are the subject of investigation in the next section.

\footnotetext{
${ }^{11}$ The proposition has been fully verified by explicitly checking all possible networks. The data used have been taken from https://users.cecs.anu.edu.au/ bdm/data/graphs.html and for the case of $n=11$ they were additionally generated by the software nauty (McKay and Piperno 2014). The author of this paper owes special gratitude to Matúš Mihalák from the Department of Data Science and Knowledge Engineering at Maastricht University for his technical assistance with this task.

${ }^{12}$ While this proposition does not appear in their work, this setup is investigated in Proposition 2 in Harmsenvan Hout et al. (2013), corresponding to their case of $\rho=1 / 2$. They observe that regular networks with degrees $d<n-1$ are not stable and networks consisting of fully connected components are stable if and only if $m \geq 4 \ell-2$ for $m$ and $\ell$ being the number of nodes in every component. Moreover, they also find that the star, circle and wheel are not stable.

${ }^{13}$ Moreover, substituting the current heuristic for the equal split used in Jackson and Wolinsky (1996) also
} 


\section{Stable Networks with Irregular Components: Simulations}

Since the model produces a plethora of possible stable networks which are not regular and have no regular components, computer simulations were employed to see the range of potential outcomes. Cycles are possible with a specific non-random matching procedure. ${ }^{14}$ One simple cycle that occurs under this protocol is described in Appendix B.3. ${ }^{15}$ However, under random matching of potential neighbours all simulations converged to a stable network. These results are investigated below with some examples which show the main observed trends.

\section{Examples}

Example 1 below gives an in-depth look into one of the simplest irregular stable networks of the model. Example 2 shows the variability of individual payoffs within the same stable network consisting of one component. Example 3 zooms out and shows that multiple components, regular and irregular, can form a stable network.

\section{Example 1. Stability and Welfare}

Consider the stable network in Figure 1a. It consists of two cliques - an upper and a lower one which are directly connected. The members of the upper clique all have degree 5, while qualitatively changes the set of stable networks that the co-author model produces, even though the payoff function it uses is different in a few dimensions from the one in this paper. Under equal split a pairwise stable network can be partitioned into fully intraconnected components with a different number of members (as specified in Proposition 4 of Jackson and Wolinsky (1996)), while the current heuristic once again produces stable networks which can consist of irregular components. One can speculate that uniform treatment like equal splitting of resources is the main force producing only regular stable networks in this case.

${ }^{14}$ Using the conventional ordering of the adjacency matrix, it is possible to find a cycle in the following way: first, check if a link can be formed. If a link is formed, then the stability of all possible links is tested (once again following the specified order). This procedure stops if no links (could be more than one) can be severed. After no more links can be cut, the check for adding links is implemented again. The procedure is repeated until no changes (adding or severing links) are possible.

${ }^{15}$ This serves as testimony that proving convergence for all cases even under random matching is not a trivial matter. The cycle illustrates that utilitarian welfare can vary in both directions as unstable networks add or sever links. This is telling since utilitarian welfare is the usual suspect for a function (similar to a potential function) a la Jackson and Watts (2001) which behaves in a predictable way, always increasing or always decreasing between two adjacent networks to show no cycles are possible in the process. Further, Hellmann and Staudigl (2014) survey different techniques which have been applied to show existence of pairwise stable networks, none of which involve properties exhibited by the current model. In particular Hellmann (2013) identifies common network properties for which there is no closed improving network cycle. One of them is ordinal convexity in own links, i.e. if a link is desirable for a player at some point, it stays desirable when adding new links. This is clearly not the case in the current setup. 
the members of the lower one have degrees 8. The payoff of all nodes in the upper clique is: ${ }^{16}$

$$
\frac{1}{\sqrt{\frac{5 * 3}{5}+\frac{5 * 2}{8}}}\left(\frac{2}{\sqrt{\frac{8}{5}+\frac{8 * 7}{8}}}+\frac{3}{\sqrt{\frac{5 * 3}{5}+\frac{5 * 2}{8}}}\right)=1.037
$$

while the nodes in the lower clique have only:

$$
\frac{1}{\sqrt{\frac{8}{5}+\frac{8 * 7}{8}}}\left(\frac{7}{\sqrt{\frac{8 * 7}{8}+\frac{8}{5}}}+\frac{1}{\sqrt{\frac{5 * 3}{5}+\frac{5 * 2}{8}}}\right)=0.979
$$

leading to overall welfare of 11.982. The network is stable and this is shown explicitly below. There are three types of links - between the nodes in the upper clique, between the ones in the lower clique and between the two cliques. Severing one of the links would make no agent(s) strictly better off. For example, cutting the link between a node in the upper clique and a node in the lower clique would bring the node in the upper clique:

$$
\frac{1}{\sqrt{\frac{4 * 3}{5}+\frac{4}{8}}}\left(\frac{1}{\sqrt{\frac{8}{4}+\frac{8 * 6}{8}+\frac{8}{7}}}+\frac{3}{\sqrt{\frac{5 * 2}{5}+\frac{5}{4}+\frac{5 * 2}{8}}}\right)=1.025<1.037
$$

and it would bring to the node in the lower clique:

$$
\frac{1}{\sqrt{\frac{7 * 7}{8}}} \frac{7}{\sqrt{\frac{8 * 6}{8}+\frac{8}{5}+\frac{8}{7}}}=0.957<0.979 .
$$

Similarly, none of the links between members of the upper clique would be cut:

$$
\frac{1}{\sqrt{\frac{4 * 2}{5}+\frac{4 * 2}{8}}}\left(\frac{2}{\sqrt{\frac{8}{4}+\frac{8 * 7}{8}}}+\frac{2}{\sqrt{\frac{5}{5}+\frac{5 * 2}{4}+\frac{5 * 2}{8}}}\right)=0.983<1.037 .
$$

None of the links between members of the lower clique would be cut:

$$
\frac{1}{\sqrt{\frac{7}{5}+\frac{7 * 6}{8}}}\left(\frac{6}{\sqrt{\frac{8 * 6}{8}+\frac{8}{5}+\frac{8}{7}}}+\frac{1}{\sqrt{\frac{5 * 3}{5}+\frac{5}{8}+\frac{5}{7}}}\right)=0.973<0.979 .
$$

Finally, the only type of link that could be formed is between the upper and lower clique. For instance, this would bring the node in the upper clique:

$$
\begin{aligned}
\frac{1}{\sqrt{\frac{6 * 3}{5}+\frac{6 * 2}{8}+\frac{6}{9}}}\left(\frac{1}{\sqrt{\frac{9}{5}+\frac{9 * 7}{8}+\frac{9}{6}}}+\frac{1}{\sqrt{\frac{5}{6}+\frac{5 * 2}{5}+\frac{5}{9}+\frac{5}{8}}}+\frac{2}{\sqrt{\frac{8}{6}+\frac{8 * 6}{8}+\frac{8}{9}}}+\frac{2}{\sqrt{\frac{5 * 2}{5}+\frac{5 * 2}{8}+\frac{5}{6}}}\right)= \\
=1.035<1.037
\end{aligned}
$$

\footnotetext{
${ }^{16}$ All numbers are rounded up to the third digit.
} 
and therefore the link would not be formed. In contrast, nodes in the lower clique can potentially benefit from this link:

$$
\begin{aligned}
\frac{1}{\sqrt{\frac{9 * 7}{8}+\frac{9}{6}+\frac{9}{5}}}\left(\frac{1}{\sqrt{\frac{6 * 3}{5}+\frac{6 * 2}{8}+\frac{6}{9}}}+\frac{1}{\sqrt{\frac{5}{6}+\frac{5 * 2}{5}+\frac{5}{9}+\frac{5}{8}}}+\frac{2}{\sqrt{\frac{8}{9}+\frac{8}{6}+\frac{8 * 6}{8}}}+\frac{5}{\sqrt{\frac{8}{9}+\frac{8}{5}+\frac{8 * 6}{8}}}\right)= \\
=0.996>0.979 .
\end{aligned}
$$

\section{Example 2. Degrees and Individual payoffs}

Figure $1 b$ once again has two cliques but in each of them some members are also connected to an intermediary node and as a result they have one degree higher than the rest (degree 6 in the case of the three nodes in the upper clique and degree 13 in the case of nodes from the lower clique). In this setting the highest payoffs 1.015 are reaped by the members of the smaller clique who are not connected to anyone else, while the intermediary has the smallest payoff 0.96. The overall welfare is 19.966 .

Figure $1 c$ shows a very similar structure, but in it the two clearly delineated cliques also have direct connections between each other. Interestingly, the node which is connected to the upper clique directly and through the intermediary has payoff 1.019, another node has two direct connections to the upper clique and the highest payoff of 1.021, while the node which is connected only through the intermediary node has payoff 0.998. The lowest payoff in the case, 0.984 is for the members of the upper clique who are not in any way connected to the lower one. Moreover, nodes with the same degree, can have different payoffs, e.g. three different nodes in the upper clique have the same degree of 12, but one of them has 0.993 , the second one has 0.984 , while the third one has 0.988 . The overall welfare is 19.968 .

Figure $1 d$ has overall welfare of 19.995. It has 13 nodes with degrees 15, 11 with degree 4, and one with degree 12,13 and 14 .

\section{Example 3. Multiple components}

Figure 2 illustrates another interesting feature of the stable networks in the model - they can consist of disconnected irregular and regular components. The irregular component in such a structure is stable on its own. However, it cannot be verified that every stable irregular component can be integrated in such a structure with multiple complete components and still stay stable. In fact Figure $1 \mathrm{~b}$ presents a counterexample. Combined with regular complete components with sizes between 6-8 and between 11-18 in one network the resulting whole in not stable. However, this is not the case for complete components with sizes between 2-5 or 9 and 10. This particular result is most likely related to the sizes of the cliques. The other subparts of the figure seem to be more resistant to such events, since combining Figures $1 a, 1 c, 1 d$ with complete regular components of sizes between 2-60 still results in stable networks. 


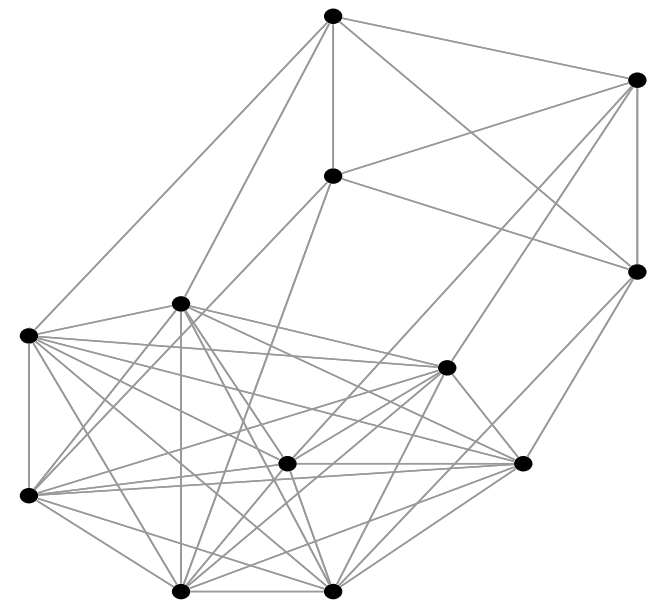

(a) The smallest simulated irregular stable network: $n=12$

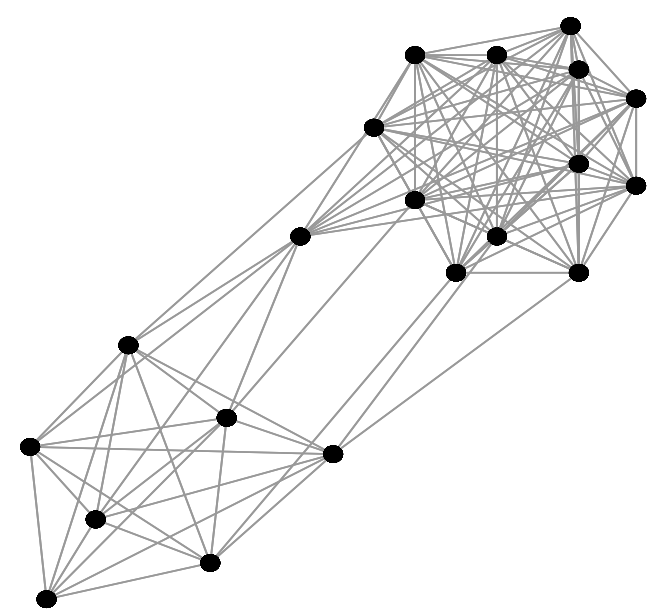

(c) Two cliques linked by an intermediary, but also with separate links: $n=20$

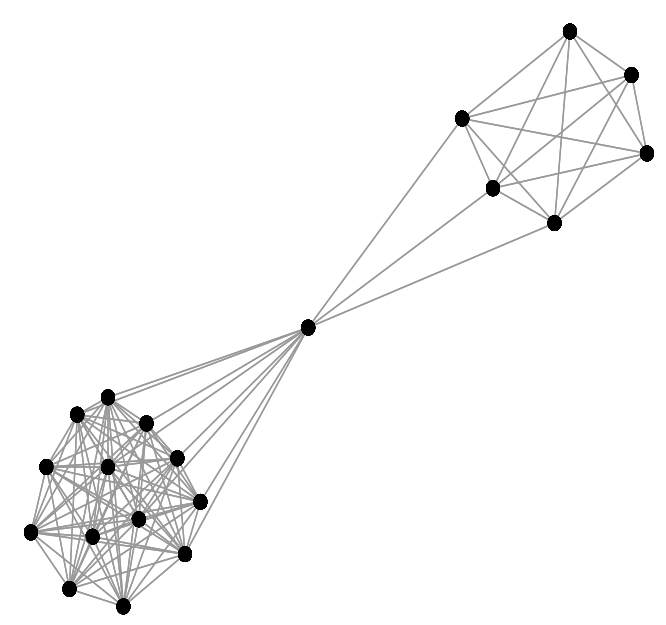

(b) Upper and lower cliques linked by an intermediary agent: $n=20$

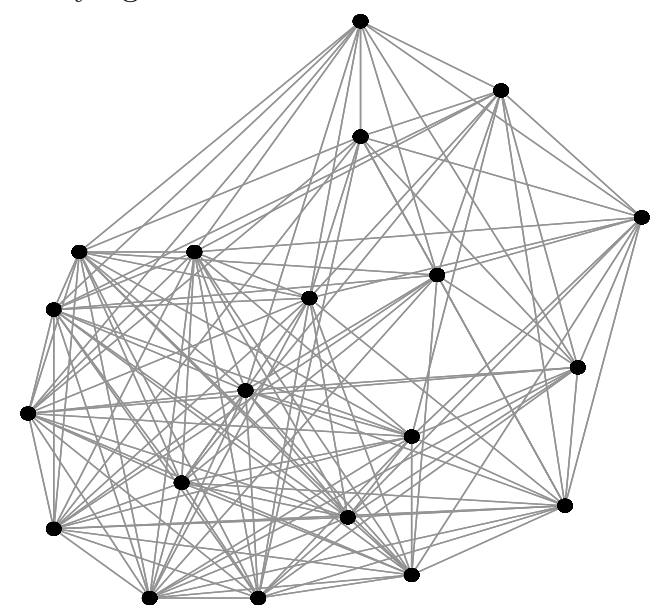

(d) A network with a core-periphery structure: $n=$ 20

Figure 1: Irregular stable networks
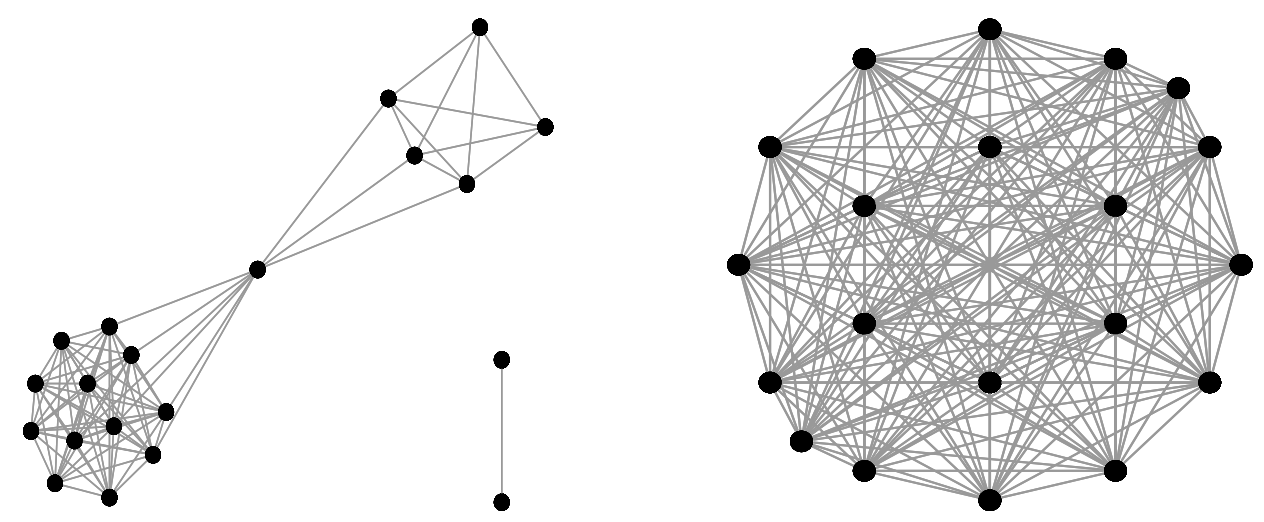

Figure 2: A stable network with regular and irregular components: $n=40$ 
As illustrated by Figure 2 stable networks can consist of one irregular component and multiple regular ones. While combining any two of the irregular networks in Figure 1 does not yield a stable network, stable networks can consist of more than one irregular component.

\section{Network Structure}

Stable networks consisting of irregular components start appearing for $n \geq 12$. The components fall into three qualitative categories: networks with cliques and direct links between them (Figure 1a, 1b), networks with cliques and intermediaries (Figure 1c) between them and networks with a core-periphery structure (Figure 1d). The cliques are reminiscent of the disjoint complete components that were described in the previous section, since they are connected relatively regularly and moreover, they are never of the same (or very similar) sizes. However, the irregular stable networks occasionally have a core and some relatively peripheral nodes like in Figure 1d.

It would be extremely unusual if this simple model perfectly reproduced all stylised facts about social networks. However, qualitatively some curious features can be identified. For example, in the simulated networks the distance between pairs of nodes in components is relatively small. The networks exhibit a high degree of clustering, high degree nodes tend to be related to other high-degree nodes and low-degree nodes also tend to be related to lower-degree nodes. There are fewer nodes with medium degrees and relatively more with high and low degrees as compared to networks where links are formed uniformly at random. These are all observed features of social networks (cf. Jackson and Rogers (2007)).

\section{Welfare}

While no formal results are derived for this, the price of anarchy in simulated pairwise stable networks is smaller than $\frac{n}{n-1}$, which implies that the loss of welfare in stable networks is not big, i.e. observing clique behaviour and even apparent separation is not necessarily significantly harming welfare and therefore might not warrant an intervention. According to the simulations the payoffs are very close to the theoretical maximum $n$, despite nonnegligible differences between nodes' payoffs. One possible explanation for this occurrence is the implicit correction for externalities inherent in the current decision rule. As a node creates another link it imposes an externality on its direct neighbours but by becoming a less-desirable neighbour it immediately attracts smaller investments. In this way it partially internalises the externality it has imposed on others. This is not a feature of the equal split rule, where one only imposes an externality on one's current neighbours by acquiring new ones. 


\section{Extensions}

It is evident that the outcomes under the current model greatly diverge from a similar setting where agents spread their resources equally between their neighbours. In fact it is possible to construe the two setups as two extreme cases, depending on how much weight (denoted by the parameter $\alpha$ ) the degree of the node's neighbours has on the node's investments: the equal split attributes no weight $(\alpha=0)$ while the current heuristic gives weight inversely proportional to the degree $(\alpha=1) \cdot{ }^{17}$ Taking this broader perspective for completeness, it is immediately possible to note some interesting outcomes. Networks consisting of complete components are stable. Importantly, the possible size of complete components within the same stable network varies depending on the particular $\alpha$. Moreover, for any fixed $n$ it seems to be possible to find an $\alpha$ close enough to 0 (but not equal) for which the stable networks would only consist of complete components. Taking the opposite perspective, for any fixed $\alpha \neq 0$ it seems to be possible to find an $n$ after which the stable networks would not consist of only complete components. ${ }^{18}$

The line, star and circle networks which were shown to be unstable in Proposition 6 and Corollary 2 (for $\alpha=1$ ) can also be shown to be unstable for the intermediate values of $\alpha$. Finally, taking values of $\alpha$ sufficiently close to 1 (bigger and smaller) results in (qualitatively) similar stable networks to the ones explored with the simulations in this section.

A natural addition to this model is to consider the case with fixed linking costs incurred for every link one makes. Clearly, if the linking costs are relatively high, the set of stable networks is going to shrink and allow only the ones with the highest welfare be stable (e.g. networks consisting of complete components). In the opposite case, for relatively low linking costs, it is possible that for a specific $n$ the set of stable networks is preserved. High and low linking costs are determined by the relative size of the network. A network with more nodes would have a lower threshold for the linking costs for which the set of stable networks is preserved compared to the case of no linking costs, and vice versa. This is due to the fact that in a network with more nodes an additional link would bring less on average (within a connected component) and compared to the fixed linking cost this would sometimes not justify creating the link. Intermediate linking costs would logically change the nature of the stable networks for any specific $n$, but it would still be possible to observe pairwise stable networks consisting or regular and irregular components.

\footnotetext{
${ }^{17}$ Appendix B.2 partially addresses this question analytically.

${ }^{18}$ One intuitive way to explain this is that the proof of Proposition 9 includes strict rather than weak inequalities in its end.
} 


\section{Conclusion}

Heuristics have been a relatively overlooked possibility for network formation, while being widely explored in other areas of economics. This paper takes first steps in this direction by investigating the possible outcomes of a simple heuristic, investing more in people who are deemed more likely to invest in you, based on their degree in the network. This is an intuitively appealing idea for modelling social value but it is also supported by experimental evidence. However, an important test for the credibility of such an approach is also considering the outcomes it produces. This is the main objective of this paper. It looked into the pairwise stable networks that emerge from applying such a heuristic. They are non-trivial and possess interesting properties. On the one hand one starting state can yield many possible outcomes. On the other hand, especially with larger networks, the outcomes frequently exhibit descriptive features that are observed in real-life social networks. They often have cliques, connected by several intermediaries, but also sometimes have a well connected core and periphery structure. It is interesting to note that while on the individual level the potential losses that are implied by following the heuristic are non-negligible, they do not result in big losses in welfare when looking at the aggregate level, since the externalities the heuristic imposes are partially internalised. It is also worth noting that it is possible for people to be in the periphery of the network and to benefit more than the ones in more central positions - having a few good friends could be better than having many superficial acquaintances. Moreover, agents with the same degree can have different payoffs in the same network i.e. the correlation between the number of connections and payoff is not one-to-one and results in a diverse set of possible outcomes. One of the most appealing features of the current setup is that the rich set of stable networks results from a minimal number of simple starting assumptions - the agents are not initially differentiated, they are matched randomly, use myopic decisions and do not calculate complicated best responses but still end up in particular identifiable structures.

Building on the current results, a promising possible extension of the model could be adding an informational dimension to it. This would undoubtedly change the set of stable networks depending on the specific payoffs from information and can be an additional robustness check for the networks identified in the current paper. Looking at an even larger set of different payoff functions for the links, e.g. functions with multiple additive elements, can give an indication to what extent the stable network structures are determined by the allocation of resources at the link level. Finally, it would be interesting to see if there are functional forms that can produce best response results (in terms of the allocation of resources) which come qualitatively close to the allocations prescribed by the heuristic in this paper. 


\section{References}

Albornoz, F., A. Cabrales, and E. Hauk (forthcoming). Occupational choice with endogenous spillovers. The Economic Journal.

Bala, V. and S. Goyal (2000). A noncooperative model of network formation. Econometrica $68(5), 1181-1229$.

Baumann, L. (2017). A model of weighted network formation. Manuscript submitted for publication.

Bloch, F. and B. Dutta (2009). Communication networks with endogenous link strength. Games and Economic Behavior 66(1), 39-56.

Bourlès, R., Y. Bramoullé, and E. Perez-Richet (2017). Altruism in networks. Econometrica $85(2), 675-689$.

Bramoullé, Y., S. Currarini, M. O. Jackson, P. Pin, and B. W. Rogers (2012). Homophily and long-run integration in social networks. Journal of Economic Theory 147(5), 1754-1786.

Brueckner, J. K. (2006). Friendship networks. Journal of Regional Science 46(5), 847-865.

Cover, T. M. and J. A. Thomas (2006). Elements of information theory (2nd ed.). A WileyInterscience publication. Hoboken, N.J.: Wiley-Interscience.

Deroian, F. (2009). Endogenous link strength in directed communication networks. Mathematical Social Sciences 57(1), 110-116.

Galeotti, A., S. Goyal, M. O. Jackson, F. Vega-Redondo, and L. Yariv (2010). Network games. The Review of Economic Studies 7r(1), 218-244.

Hämäläinen, R. P., J. Luoma, and E. Saarinen (2013). On the importance of behavioral operational research: The case of understanding and communicating about dynamic systems. European Journal of Operational Research 228(3), 623-634.

Harmsen-van Hout, M. J. W., B. G. C. Dellaert, and P. J.-J. Herings (2016). Heuristic decision making in network linking. European Journal of Operational Research 251(1), 158-170.

Harmsen-van Hout, M. J. W., P. J.-J. Herings, and B. G. C. Dellaert (2013). Communication network formation with link specificity and value transferability. European Journal of Operational Research 229(1), 199-211.

Hellmann, T. (2013). On the existence and uniqueness of pairwise stable networks. International Journal of Game Theory 42(1), 211-237. 
Hellmann, T. and M. Staudigl (2014). Evolution of social networks. European Journal of Operational Research 234(3), 583-596.

Jackson, M. O. and B. W. Rogers (2007). Meeting strangers and friends of friends: How random are social networks? The American Economic Review 97(3), 890-915.

Jackson, M. O. and A. Watts (2001). The existence of pairwise stable networks. Seoul Journal of Economics $14(3)$.

Jackson, M. O. and A. Wolinsky (1996). A strategic model of social and economic networks. Journal of Economic Theory 71(1), 44-74.

Kováŕík, J., F. Mengel, and J. G. Romero (2018). Learning in network games. Quantitative Economics 9(1), 85-139.

McKay, B. D. and A. Piperno (2014). Practical graph isomorphism, \{II $\}$. Journal of Symbolic Computation 60(0), $94-112$.

McPherson, M., L. Smith-Lovin, and J. M. Cook (2001). Birds of a feather: Homophily in social networks. Annual Review of Sociology 27(1), 415-444.

Morbitzer, D., V. Buskens, S. Rosenkranz, and W. Raub (2014). How farsightedness affects network formation. Analyse und Kritik 36(1), 103.

Salonen, H. (2016). Reciprocal equilibria in link formation games. The Czech Economic Review 9(3), 169-183.

So, C. (2016). Network formation with endogenous link strength and decreasing returns to investment. Games 7(4). 


\section{A Proofs}

Proof of Proposition 5: To prove the statement it is sufficient to show that in any regular network with degree $k$ which is not complete two of its nodes would want to form a connection. This is equivalent to the condition:

$$
\frac{1}{\sqrt{\frac{k+1}{k+1}+k \frac{k+1}{k}}}\left(\frac{1}{\sqrt{\frac{k+1}{k+1}+k \frac{k+1}{k}}}+\frac{\alpha}{\sqrt{2 \frac{k}{k+1}+(k-2) \frac{k}{k}}}+\frac{k-\alpha}{\sqrt{\frac{k}{k+1}+(k-1) \frac{k}{k}}}\right)>1
$$

where $\alpha \geq 1$ is the number of agents that are mutual neighbours of the two connecting nodes. The condition can be simplified.

$$
\frac{1}{\sqrt{k+2}}\left(\frac{1}{\sqrt{k+2}}+\frac{\alpha \sqrt{k+1}}{\sqrt{k^{2}+k-2}}+\frac{(k-\alpha) \sqrt{k+1}}{\sqrt{k^{2}+k-1}}\right)>1
$$

Since

$$
\frac{\sqrt{k+1}}{\sqrt{k^{2}+k-2}} \geq \frac{\sqrt{k+1}}{\sqrt{k^{2}+k-1}}
$$

holds for every $k \geq 1$, taking $\alpha=0$ presents the worst-case scenario for Inequality (1), i.e. the case in which its left-hand side has the lowest possible value, presenting the lowest incentive for the two nodes to connect. This leaves:

$$
\begin{gathered}
\frac{1}{\sqrt{k+2}}\left(\frac{1}{\sqrt{k+2}}+\frac{k \sqrt{k+1}}{\sqrt{k^{2}+k-1}}\right)>1 \Leftrightarrow \frac{1}{k+2}+\frac{k \sqrt{k+1}}{\sqrt{\left(k^{2}+k-1\right)(k+2)}}>1 \\
\frac{k \sqrt{k+1}}{\sqrt{\left(k^{2}+k-1\right)(k+2)}}>\frac{k+1}{k+2} \\
k \sqrt{k+2}>\sqrt{(k+1)\left(k^{2}+k-1\right)} \Leftrightarrow k^{3}+2 k^{2}>k^{3}+k^{2}-k+k^{2}+k-1
\end{gathered}
$$

which is always true for $k \geq 3$.

Proof of Proposition 6: This proof contains four parts.

A) Line: The two ends would want to connect, since:

$$
\frac{1}{\sqrt{\frac{1}{2}}} \frac{1}{\sqrt{\frac{2}{1}+\frac{2}{1}}}<1 ; \frac{1}{\sqrt{\frac{1}{2}}} \frac{1}{\sqrt{\frac{2}{1}+\frac{2}{2}}}<1
$$

The first inequality refers to a line of length 3 , while the second one covers all cases of longer lines.

B) Star: It is sufficient to show that two of the periphery nodes of a star network with $n \geq 3$ would want to form a link. The condition is:

$$
\frac{1}{\sqrt{\frac{2}{n-1}+\frac{2}{2}}}\left(\frac{1}{\sqrt{\frac{n-1}{2} 2+\frac{n-1}{1}(n-3)}}+\frac{1}{\sqrt{\frac{2}{n-1}+\frac{2}{2}}}\right) \geq \frac{1}{\sqrt{\frac{1}{n-1}}} \frac{1}{\sqrt{(n-1) \frac{n-1}{1}}}
$$


Simplified:

$$
\begin{gathered}
\frac{1}{\sqrt{\frac{2}{n-1}+1}}\left(\frac{1}{\sqrt{n-1+(n-1)(n-3)}}+\frac{1}{\sqrt{\frac{2}{n-1}+1}}\right) \geq \frac{1}{\sqrt{n-1}} \\
\frac{1}{\sqrt{\frac{n+1}{n-1}}}\left(\frac{1}{\sqrt{(n-1)(n-2)}}+\frac{1}{\sqrt{\frac{n+1}{n-1}}}\right) \geq \frac{1}{\sqrt{n-1}} \Leftrightarrow \frac{1}{\sqrt{(n+1)(n-2)}}+\frac{n-1}{n+1} \geq \frac{1}{\sqrt{n-1}} \\
\frac{\sqrt{n-1}}{\sqrt{(n+1)(n-2)}}+\frac{(n-1) \sqrt{n-1}}{n+1} \geq 1
\end{gathered}
$$

$\frac{(n-1) \sqrt{n-1}}{n+1}$ is bigger than 1 for $n \geq 4$ and it has a positive first derivative. The first term of (2) is always positive. Finally, a specific check for $n=3$ shows that (2) holds for integers $n \geq 3$.

C) Wheel: Here it is sufficient to prove that at least one node would want to disconnect from the centre. Taking a peripheral node the following inequality should hold:

$$
\frac{1}{\sqrt{2 * \frac{3}{3}+\frac{3}{n}}}\left(\frac{2}{\sqrt{2 * \frac{3}{3}+\frac{3}{n}}}+\frac{1}{\sqrt{n \frac{n}{3}}}\right)<\frac{1}{\sqrt{2 * \frac{2}{3}}} \frac{2}{\sqrt{\frac{3}{2}+\frac{3}{3}+\frac{3}{n}}}
$$

where the right-hand side expresses the payoff of disconnecting from the centre. Simplifying:

$$
\begin{gathered}
\frac{1}{\sqrt{\frac{2 n+3}{n}}}\left(\frac{2}{\sqrt{\frac{2 n+3}{n}}}+\frac{\sqrt{3}}{n}\right)<\frac{\sqrt{3}}{\sqrt{\frac{5}{2}+\frac{3}{n}}} \Leftrightarrow \frac{1}{\sqrt{\frac{2 n+3}{n}}}\left(\frac{2}{\sqrt{\frac{2 n+3}{n}}}+\frac{\sqrt{3}}{n}\right)<\frac{\sqrt{3}}{\sqrt{\frac{5 n+6}{2 n}}} \\
\frac{2 n}{2 n+3}+\frac{\sqrt{3}}{\sqrt{n(2 n+3)}}<\sqrt{\frac{6 n}{5 n+6}}
\end{gathered}
$$

The right-hand side of condition (4) is greater than or equal to 1 for $n \geq 6$. The left-hand side is strictly smaller than 1 in this range of values for $n$ :

$$
\begin{gathered}
\frac{2 n}{2 n+3}+\frac{\sqrt{3}}{\sqrt{n(2 n+3)}}<1 \Leftrightarrow \frac{\sqrt{3}}{\sqrt{n(2 n+3)}}<\frac{3}{2 n+3} \Leftrightarrow \frac{1}{\sqrt{n}}<\frac{\sqrt{3}}{\sqrt{2 n+3}} \\
2 n+3<3 n
\end{gathered}
$$

Therefore, condition (3) holds for $n \geq 6$ and in these cases the wheel is not stable. To show that when $n \in\{4,5\}$ the wheel is also not stable, the conditions for two peripheral nodes to connect are checked separately. For $n=4$ :

$$
\begin{aligned}
\frac{1}{\sqrt{2 * \frac{3}{3}+\frac{3}{n}}}\left(\frac{2}{\sqrt{2 * \frac{3}{3}+\frac{3}{n}}}+\frac{1}{\sqrt{n \frac{n}{3}}}\right)< \\
\\
\frac{1}{\sqrt{\frac{4}{n}+2 * \frac{4}{3}+\frac{4}{4}}}\left(\frac{1}{\sqrt{\frac{4}{n}+2 * \frac{4}{3}+\frac{4}{4}}}+\frac{1}{\sqrt{2 * \frac{n}{4}+(n-2) \frac{n}{3}}}+\frac{2}{\sqrt{2 * \frac{3}{4}+\frac{3}{n}}}\right)
\end{aligned}
$$


And for $n=5$ :

$$
\begin{aligned}
& \frac{1}{\sqrt{2 * \frac{3}{3}+\frac{3}{n}}}\left(\frac{2}{\sqrt{2 * \frac{3}{3}+\frac{3}{n}}}+\frac{1}{\sqrt{n \frac{n}{3}}}\right)< \\
& \frac{1}{\sqrt{\frac{4}{n}+2 * \frac{4}{3}+\frac{4}{4}}}\left(\frac{1}{\sqrt{\frac{4}{n}+2 * \frac{4}{3}+\frac{4}{4}}}+\frac{1}{\sqrt{2 * \frac{n}{4}+(n-2) \frac{n}{3}}}+\frac{1}{\sqrt{2 * \frac{3}{4}+\frac{3}{n}}}+\frac{1}{\sqrt{\frac{3}{n}+\frac{3}{4}+\frac{3}{3}}}\right)
\end{aligned}
$$

Both conditions hold for the specific values of $n$. Therefore, the wheel with $n \geq 4$ in the periphery is not stable.

D) This proof will be presented in two parts - D1) which deals with complete biregular graphs and D2) which deal with incomplete biregular graphs.

D1) In a bipartite graph with $m$ and $k=n-m$ nodes in the two sets such that $m \leq k$ at least one set of nodes would want to connect and therefore the network would not be stable, since the payoff of a connection between two nodes in the $m$-set would be always preferred to the status quo:

$$
\frac{1}{\sqrt{\frac{m^{2}}{k}}} * \frac{m}{\sqrt{\frac{k^{2}}{m}}} \leq \frac{1}{\sqrt{\frac{m(m+1)}{k}+\frac{m+1}{m+1}}}\left(\frac{1}{\sqrt{\frac{m(m+1)}{k}+\frac{m+1}{m+1}}}+\frac{m}{\sqrt{\frac{k(k-2)}{m}+\frac{2 k}{m+1}}}\right)
$$

Simplify:

$$
\begin{gathered}
\frac{1}{\sqrt{\frac{(m+1) m+n}{k}}}\left(\frac{1}{\sqrt{\frac{(m+1) m+k}{k}}}+\frac{m}{\sqrt{\frac{k(k-2)(m+1)+2 m k}{m(m+1)}}}\right) \geq \sqrt{\frac{m}{k}} \\
\frac{k}{(m+1) m+k}+\frac{m \sqrt{m(m+1)}}{\sqrt{((m+1) m+k)((k-2)(m+1)+2 m)}} \geq \sqrt{\frac{m}{k}} \\
n \sqrt{k((k-2)(m+1)+2 m)}+m \sqrt{m k(m+1)((m+1) m+k)} \geq((m+1) m+k) \sqrt{m((k-2)(m+1)+2 m)} \\
k \sqrt{k(m k+k-2)}+m \sqrt{m k(m+1)\left(m^{2}+m+k\right)} \geq\left(m^{2}+m+n\right) \sqrt{m(m k+k-2)} \\
k \sqrt{k(m k+k-2)} \geq \sqrt{m^{2}+m+k}\left(\sqrt{m\left(m^{2}+m+k\right)(m k+k-2)}-m \sqrt{m k(m+1)}\right)
\end{gathered}
$$

Take the first part of the left-hand side and the second part of the right-hand side.

$$
k \sqrt{k} \geq \sqrt{m\left(m^{2}+m+k\right)(m k+k-2)}-m \sqrt{m k(m+1)}
$$

Rearrange:

$$
k \sqrt{k}+m \sqrt{m k(m+1)} \geq \sqrt{m\left(m^{2}+m+k\right)(m k+k-2)}
$$

Square both sides of the condition.

$$
\begin{gathered}
k^{3}+2 k^{2} m \sqrt{m(m+1)}+m^{3} k(m+1) \geq\left(m^{2}(m+1)+k m\right)(k(m+1)-2) \\
k^{3}+2 k^{2} m \sqrt{m(m+1)}+m^{3} k(m+1) \geq k m^{2}(m+1)^{2}+k^{2} m(m+1)-2 m^{2}(m+1)-2 k m
\end{gathered}
$$




$$
\begin{gathered}
k^{3}+2 k^{2} m \sqrt{m(m+1)}+m^{2} k(m+1)(m-m-1) \geq k^{2} m(m+1)-2 m^{2}(m+1)-2 k m \\
k^{3}+2 m^{2}(m+1)+2 k m+2 k^{2} m \sqrt{m(m+1)}-m^{2} k(m+1)-k^{2} m(m+1) \geq 0
\end{gathered}
$$

Consider only the last three terms (the first of them is split) $k^{2} m \sqrt{m(m+1)}-m^{2} k(m+1)+$ $k^{2} m \sqrt{m(m+1)}-k^{2} m(m+1)$. Each of them is positive separately, except for $k=m+1$. This requires a separate check.

Now take the second part of the left-hand side and the first part of the right-hand side.

$$
\sqrt{m k+k-2} \geq \sqrt{m^{2}+m+k}
$$

Inequality (6) is true for $k \geq m+1+\frac{2}{m}$, so what is left is to see that condition (5) holds for $m \in\{k, k-1, k-2\}$. A manual check shows this is also true. Therefore a complete bipartite graph is not stable in this setting.

D2) In a biregular graph which is not complete at least two different nodes which are not in the same subgroup of nodes (with degrees $k$ and $\ell$ ) would want to connect.

$$
\frac{1}{\sqrt{\frac{k+1}{\ell+1}+\frac{(k+1) k}{\ell}}}\left(\frac{1}{\sqrt{\frac{\ell+1}{k+1}+\frac{(\ell+1) \ell}{k}}}+\frac{k}{\sqrt{\frac{\ell(\ell-1)}{k}+\frac{\ell}{k+1}}}\right) \geq \frac{1}{\sqrt{\frac{k * k}{\ell}}} \frac{k}{\sqrt{\frac{\ell * \ell}{k}}}
$$

Simplify:

$$
\begin{gathered}
\frac{1}{\sqrt{\frac{(k+1)(\ell+k \ell+k)}{(\ell+1) \ell}}}\left(\frac{1}{\sqrt{\frac{(\ell+1)(\ell+k \ell+k)}{(k+1) k}}}+\frac{k}{\sqrt{\frac{\ell(k \ell+\ell-1)}{k(k+1)}}}\right) \geq \sqrt{\frac{k}{\ell}} \\
\frac{\sqrt{\ell k}}{\ell+k \ell+k}+\frac{k \sqrt{k(\ell+1)}}{\sqrt{(\ell+k \ell+k)(k \ell+\ell-1)}} \geq \sqrt{\frac{k}{\ell}} \\
\frac{\ell}{\ell+k \ell+k}+\frac{k \sqrt{\ell(\ell+1)}}{\sqrt{(\ell+k \ell+k)(k \ell+\ell-1)}} \geq 1 \Leftrightarrow \frac{k \sqrt{\ell(\ell+1)}}{\sqrt{(\ell+k \ell+k)(k \ell+\ell-1)}} \geq \frac{k \ell+k}{\ell+k \ell+k} \\
\frac{\sqrt{\ell}}{\sqrt{k \ell+\ell-1}} \geq \frac{\sqrt{\ell+1}}{\sqrt{\ell+k \ell+k}} \Leftrightarrow \frac{\ell}{k \ell+\ell-1} \geq \frac{\ell+1}{\ell+k \ell+k} \\
\ell(\ell+k \ell+k) \geq \ell(k \ell+\ell-1)+k \ell+\ell-1 \Leftrightarrow \ell(k+1) \geq k \ell+\ell-1
\end{gathered}
$$

This is always true.

Proof of Proposition 7: As already showed in Proposition 3 no node in a complete graph would disconnect from the rest. Therefore, in order to prove the statement it is sufficient to show that the two subgraphs would not form links for $m_{1} \geq m_{2}+2$ (here it is assumed that $m_{1}>m_{2}$ without loss of generality). The conditions for two nodes from the subgraphs to want to form a link are:

$$
\frac{1}{\sqrt{\frac{m_{2}}{m_{1}}+\frac{\left(m_{2}-1\right) m_{2}}{m_{2}-1}}}\left(\frac{1}{\sqrt{\frac{m_{1}}{m_{2}}+\frac{\left(m_{1}-1\right) m_{1}}{m_{1}-1}}}+\frac{m_{2}-1}{\sqrt{\frac{m_{2}-1}{m_{2}}+\frac{\left(m_{2}-2\right)\left(m_{2}-1\right)}{m_{2}-1}}}\right) \geq 1
$$




$$
\frac{1}{\sqrt{\frac{m_{1}}{m_{2}}+\frac{\left(m_{1}-1\right) m_{1}}{m_{1}-1}}}\left(\frac{1}{\sqrt{\frac{m_{2}}{m_{1}}+\frac{\left(m_{2}-1\right) m_{2}}{m_{2}-1}}}+\frac{m_{1}-1}{\sqrt{\frac{m_{1}-1}{m_{1}}+\frac{\left(m_{1}-2\right)\left(m_{1}-1\right)}{m_{1}-1}}}\right) \geq 1
$$

where one of them needs to hold strictly. They could be simplified to:

$$
\begin{aligned}
& \frac{1}{\sqrt{\frac{m_{2}\left(m_{1}+1\right)}{m_{1}}}}\left(\frac{1}{\sqrt{\frac{m_{1}\left(m_{2}+1\right)}{m_{2}}}}+\frac{m_{2}-1}{\sqrt{\frac{m_{2}^{2}-m_{2}-1}{m_{2}}}}\right) \geq 1 \\
& \frac{1}{\sqrt{\frac{m_{1}\left(m_{2}+1\right)}{m_{2}}}}\left(\frac{1}{\sqrt{\frac{m_{2}\left(m_{1}+1\right)}{m_{1}}}}+\frac{m_{1}-1}{\sqrt{\frac{m_{1}^{2}-m_{1}-1}{m_{1}}}}\right) \geq 1
\end{aligned}
$$

The first parts of the expressions on the left-hand side of the inequalities, $\frac{1}{\sqrt{\frac{m_{1}\left(m_{2}+1\right)}{m_{2}}}} \frac{1}{\sqrt{\frac{m_{2}\left(m_{1}+1\right)}{m_{1}}}}$, are the same. Comparing the second parts, it is true that:

$$
\frac{\left(m_{2}-1\right) \sqrt{m_{1}}}{\sqrt{\left(m_{1}+1\right)\left(m_{2}^{2}-m_{2}-1\right)}} \geq \frac{\left(m_{1}-1\right) \sqrt{m_{2}}}{\sqrt{\left(m_{2}+1\right)\left(m_{1}^{2}-m_{1}-1\right)}}
$$

because, for $m_{1} \geq m_{2}$, it always holds that:

$$
\frac{\sqrt{m_{1}\left(m_{1}^{2}-m_{1}-1\right)}}{\left(m_{1}-1\right) \sqrt{m_{1}+1}} \geq \frac{\sqrt{m_{2}\left(m_{2}^{2}-m_{2}-1\right)}}{\left(m_{2}-1\right) \sqrt{m_{2}+1}} .
$$

Therefore, condition (8) is binding and it is sufficient to show that it holds for both (7) and (8) to hold. For $m_{1} \geq 4$, condition (8) is equivalent to:

$$
\begin{gathered}
\sqrt{m_{1}^{2}-m_{1}-1}+\left(m_{1}-1\right) \sqrt{m_{2}\left(m_{1}+1\right)} \geq \sqrt{\left(m_{1}+1\right)\left(m_{2}+1\right)\left(m_{1}^{2}-m_{1}-1\right)} \\
m_{1}^{2}-m_{1}-1+m_{2}\left(m_{1}-1\right)^{2}\left(m_{1}+1\right)+2\left(m_{1}-1\right) \sqrt{m_{2}\left(m_{1}+1\right)\left(m_{1}^{2}-m_{1}-1\right)} \geq\left(m_{1}+1\right)\left(m_{2}+1\right)\left(m_{1}^{2}-m_{1}-1\right) \\
m_{2}\left(m_{1}^{3}-m_{1}^{2}-m_{1}-1-m_{1}^{3}+2 m_{1}+1\right)+2 \sqrt{m_{2}\left(m_{1}+1\right)\left(m_{1}^{2}-m_{1}-1\right)}\left(m_{1}-1\right)-m_{1}\left(m_{1}^{2}-m_{1}-1\right) \geq 0
\end{gathered}
$$

This yields: $\frac{m_{1}^{2}-m_{1}-1}{m_{1}+1} \leq m_{2} \leq \frac{m_{1} \sqrt{\left(m_{1}+1\right)\left(m_{1}^{2}-m_{1}-1\right)}}{\left(m_{1}-2\right)\left(m_{1}+1\right)}$, but since $m_{1} \geq m_{2}$, it simplifies to $\frac{m_{1}^{2}-m_{1}-1}{m_{1}+1} \leq m_{2} \leq m_{1}$ or $m_{1}-1-\frac{m_{1}}{m_{1}+1} \leq m_{2} \leq m_{1}$. Therefore, conditions (7) and (8) hold for only $m_{2} \in\left\{m_{1}-1, m\right\}$.

Proof of Proposition 9: It is sufficient to show that any other networks would have (at least) two nodes which would always want to make a connection. The strategy in this proof is excluding all networks in which it is clear that there are two nodes which want to form a connection and considering what this implies for all other networks.

The payoff of a node $i$ with $N_{i}$ being the set of its neighbours and $d_{i}$ being its degree can be expressed and rearranged in the following way:

$$
u_{i}=\sum_{j \in N_{i}} \sqrt{\frac{1}{d_{i}} * \frac{1}{d_{j}}}=\sum_{j \in N_{i}} \frac{1}{\sqrt{d_{i} * d_{j}}}=\frac{1}{\sqrt{d_{i}}}\left(\sum_{j \in N_{i}} \frac{1}{\sqrt{d_{j}}}\right) .
$$


In order for two nodes $i$ and $k$ to want to connect the following inequalities should hold with at least one of them being strict.

$$
\begin{aligned}
& \frac{1}{\sqrt{d_{i}}}\left(\sum_{j \in N_{i}} \frac{1}{\sqrt{d_{j}}}\right) \leq \frac{1}{\sqrt{d_{i}+1}}\left(\sum_{j \in N_{i}} \frac{1}{\sqrt{d_{j}}}+\frac{1}{\sqrt{d_{k}+1}}\right) \\
& \frac{1}{\sqrt{d_{k}}}\left(\sum_{j \in N_{k}} \frac{1}{\sqrt{d_{j}}}\right) \leq \frac{1}{\sqrt{d_{k}+1}}\left(\sum_{j \in N_{k}} \frac{1}{\sqrt{d_{j}}}+\frac{1}{\sqrt{d_{i}+1}}\right)
\end{aligned}
$$

Note that $d_{i}$ and $N_{i}$ refer to the situation before a connection has been made and so $k \notin N_{i}$. Inequality (9) expresses that agent $i$ would be better off connecting to agent $k$, because his current payoff (left-hand side) is smaller than the payoff he would have if agent $k$ was his direct neighbour. In this case (right-hand side) he would split his resources in $d_{i}+1$ equal parts and get $1 /\left(d_{k}+1\right)$ of $k$ 's resources as investment. ${ }^{19}$ Consider inequality (9), which can be rewritten as:

$$
\begin{gathered}
\left(\frac{\sqrt{d_{i}+1}-\sqrt{d_{i}}}{\sqrt{d_{i}} * \sqrt{d_{i}+1}}\right)\left(\sum_{j \in N_{i}} \frac{1}{\sqrt{d_{j}}}\right)=\left(\frac{1}{\sqrt{d_{i}}}-\frac{1}{\sqrt{d_{i}+1}}\right)\left(\sum_{j \in N_{i}} \frac{1}{\sqrt{d_{j}}}\right) \leq \frac{1}{\sqrt{d_{i}+1}} * \frac{1}{\sqrt{d_{k}+1}} \\
\sum_{j \in N_{i}} \sqrt{\frac{d_{k}+1}{d_{j}}} \leq \frac{\sqrt{d_{i}}}{\sqrt{d_{i}+1}-\sqrt{d_{i}}} \Leftrightarrow \sum_{j \in N_{i}} \sqrt{\frac{d_{k}+1}{d_{j}}} \leq \sqrt{d_{i}}\left(\sqrt{d_{i}+1}+\sqrt{d_{i}}\right) \Leftrightarrow \\
\sum_{j \in N_{i}} \sqrt{\frac{d_{k}+1}{d_{j}}} \leq d_{i}+\sqrt{d_{i}\left(d_{i}+1\right)}
\end{gathered}
$$

Clearly, inequality (10) can be rewritten in a similar fashion.

Lemma 2. In a stable network if nodes $i, x$ and $k$ are such that $d_{i} \leq d_{k}, d_{x} \leq d_{k}$ and $i \in N_{k}$, then node $i$ wants to connect to node $x$.

Proof of Lemma 2: In a stable network if $i$ is connected to $k$ this implies that it also wants to be connected to it, otherwise the network would not be stable. Here it is useful to distinguish two cases: (i) $d_{x}<d_{k}$ and (ii) $d_{x}=d_{k}$.

Since the network is stable $i$ does not want to delete the link with $k$, so:

$$
\sum_{j \in N_{i}} \sqrt{\frac{d_{k}}{d_{j}}} \leq d_{i}-1+\sqrt{d_{i}\left(d_{i}-1\right)}
$$

In case $d_{x}<d_{k}$, Inequality (12) implies:

$$
\sum_{j \in N_{i}} \sqrt{\frac{d_{x}+1}{d_{j}}} \leq \sum_{j \in N_{i}} \sqrt{\frac{d_{k}}{d_{j}}} \leq d_{i}-1+\sqrt{d_{i}\left(d_{i}-1\right)}
$$

\footnotetext{
${ }^{19}$ Inequality (10) expresses the analogous idea for agent $k$ connecting to agent $i$.
} 
Excluding the middle part and adding $\sqrt{\frac{d_{x}+1}{d_{k}}} \leq 1$ on both sides implies: ${ }^{20}$

$$
\begin{gathered}
\sum_{j \in N_{i}} \sqrt{\frac{d_{x}+1}{d_{j}}}+\sqrt{\frac{d_{x}+1}{d_{k}}} \leq d_{i}-1+\sqrt{d_{i}\left(d_{i}-1\right)}+\sqrt{\frac{d_{x}+1}{d_{k}}}<d_{i}+\sqrt{d_{i}\left(d_{i}+1\right)} \\
\sum_{j \in N_{i} \cup\{k\}} \sqrt{\frac{d_{x}+1}{d_{j}}}<d_{i}+\sqrt{d_{i}\left(d_{i}+1\right)}
\end{gathered}
$$

Therefore, $i$ is willing to connect to $x$.

In case $d_{x}=d_{k}$, multiply both sides of Inequality (12) with $\sqrt{\frac{d_{k}+1}{d_{k}}}>1$ to get:

$$
\begin{gathered}
\sum_{j \in N_{i}} \sqrt{\frac{d_{k}+1}{d_{j}}} \leq d_{i} \sqrt{\frac{d_{k}+1}{d_{k}}}-\sqrt{\frac{d_{k}+1}{d_{k}}}+\sqrt{\frac{d_{i}\left(d_{i}-1\right)\left(d_{k}+1\right)}{d_{k}}} \\
\sum_{j \in N_{i}} \sqrt{\frac{d_{k}+1}{d_{j}}}+\sqrt{\frac{d_{k}+1}{d_{k}}} \leq d_{i} \sqrt{\frac{d_{k}+1}{d_{k}}}+\sqrt{\frac{d_{i}\left(d_{i}-1\right)\left(d_{k}+1\right)}{d_{k}}} \\
\sum_{j \in N_{i} \cup\{k\}} \sqrt{\frac{d_{x}+1}{d_{j}}}=\sum_{j \in N_{i}} \sqrt{\frac{d_{k}+1}{d_{j}}}+\sqrt{\frac{d_{k}+1}{d_{k}}} \leq d_{i} \sqrt{\frac{d_{k}+1}{d_{k}}}+\sqrt{\frac{d_{i}\left(d_{i}-1\right)\left(d_{k}+1\right)}{d_{k}}}
\end{gathered}
$$

In order for $i$ to want to connect to $x$, the following is sufficient for the right-hand side:

$$
\begin{gathered}
d_{i} \sqrt{\frac{d_{k}+1}{d_{k}}}+\sqrt{\frac{d_{i}\left(d_{i}-1\right)\left(d_{k}+1\right)}{d_{k}}}<d_{i}+\sqrt{d_{i}\left(d_{i}+1\right)} \\
\sqrt{d_{i}} \sqrt{\frac{d_{k}+1}{d_{k}}}\left(\sqrt{d_{i}}+\sqrt{d_{i}-1}\right)<\sqrt{d_{i}}\left(\sqrt{d_{i}}+\sqrt{d_{i}+1}\right) \\
\left(\sqrt{d_{i}}+\sqrt{d_{i}-1}\right) \sqrt{d_{k}+1}<\sqrt{d_{k}}\left(\sqrt{d_{i}}+\sqrt{d_{i}+1}\right) \\
\sqrt{d_{i}\left(d_{k}+1\right)}+\sqrt{\left(d_{i}-1\right)\left(d_{k}+1\right)}<\sqrt{d_{k}\left(d_{i}+1\right)}+\sqrt{d_{i} d_{k}} \\
\sqrt{d_{i} d_{k}+d_{i}}+\sqrt{d_{i} d_{k}-d_{k}+d_{i}-1}<\sqrt{d_{i} d_{k}+d_{k}}+\sqrt{d_{i} d_{k}}
\end{gathered}
$$

The condition holds since $d_{k} \geq d_{i} \geq 1$. Therefore, Inequality (13) is equivalent to Condition (11) and $i$ wants to connect to $x$ in both cases outlined above.

Lemma 3. In a stable network if nodes $i, x$ and $k$ are such that $d_{i} \leq d_{k}, d_{x} \leq d_{k}, i \in N_{k}$ and $x \in N_{k}$, then $x \in N_{i}$.

\footnotetext{
${ }^{20}$ The strict inequality comes from the change of signs under the square root.
} 
Proof of Lemma 3: By Lemma 2 if $k$ has a neighbour $i$ with $d_{i} \leq d_{k}$, that means $i$ is willing to connect to all nodes with degrees $\leq d_{k}$, which includes $x$. By the same token, $x$ wants to be connected to all nodes with degrees $\leq d_{k}$, which includes $i$. Therefore, in a stable network all neighbours $x$ of $k$ with $d_{x} \leq d_{k}$ will be also $i$ 's neighbours.

By Lemma 3 in a stable network all neighbours of the node with the highest degree within a component, say $h$, form a clique, i.e. they are fully interconnected and connected to $h$. Hence, they must all have degrees $\geq d_{h}$. They cannot have degrees strictly bigger than $d_{h}$, because that would contradict the assumption that $d_{h}$ has the highest degree in the component. Therefore, they must have equal degrees. In other words, in a stable network any component forms a regular subgraph. Moreover, the subgraphs are complete as every node is connected to all other nodes in the component as per Lemma 3. 


\section{B Supplementary Materials}

\section{B.1 Supplementary Materials - Proofs}

Proof of Proposition 4: To prove that a fully connected network is reachable starting from an empty graph with $n$ nodes it is sufficient to show that:

(i) it can form a complete subgraph with $n \geq m \geq 2$ nodes;

(ii) that a new node could be connected to it;

(iii) when a new node is added to the complete subgraph it triggers a process leading to a new complete (sub)graph with $m+1$ nodes.

(iv) the process is repeated until $m=n$.

Step (i) follows directly for $m=2$ since any two loose nodes prefer to be connected to being isolated. Step (ii) implicitly includes two conditions:

(a) at least one node from the complete subgraph wants to connect to one of the isolated nodes;

(b) the isolated node should also be willing to create the link.

As noted above, isolated nodes are always willing to form a link as any link brings them more than 0 . Condition (a) is captured in (14) below.

$$
\frac{1}{\sqrt{m \frac{m-1}{m-1}+\frac{m}{1}}}\left(\frac{m-1}{\sqrt{\frac{m-1}{m}+(m-2) \frac{m-1}{m-1}}}+\frac{1}{\sqrt{\frac{1}{m}}}\right)>1
$$

Simplified:

$$
\begin{gathered}
\frac{1}{\sqrt{2 m}}\left(\frac{m-1}{\sqrt{\frac{m-1+m^{2}-2 m}{m}}}+\sqrt{m}\right)>1 \Leftrightarrow \frac{m-1}{\sqrt{2\left(m^{2}-m-1\right)}}+\frac{1}{\sqrt{2}}>1 \\
m-1+\sqrt{m^{2}-m-1}>\sqrt{2\left(m^{2}-m-1\right)} \Leftrightarrow m-1>\sqrt{m^{2}-m-1}(\sqrt{2}-1) \\
m^{2}-2 m+1>\left(m^{2}-m-1\right)(3-2 \sqrt{2}) \Leftrightarrow m^{2}(2 \sqrt{2}-2)+m(1-2 \sqrt{2)}-2 \sqrt{2}+4>0
\end{gathered}
$$

This is always true for $m \geq 2$ because the discriminant of the left-hand side is negative. Hence, step (ii) is always possible.

Step (iii) also includes two parts:

(a) at least one node from the formerly complete subgraph of $m$ nodes wants to connect the formerly isolated node. This is captured in condition (16) where $c$ refers to the number of nodes in the complete graph that are connected to the formerly isolated node. 
(b) the formerly isolated node should also be willing to create the link. This is captured in condition (15) below.

$$
\frac{1}{\sqrt{\frac{(c+1)(c+1)}{m}}} \frac{c+1}{\sqrt{\frac{m}{c+1}+\frac{m(m-c-1)}{m-1}+\frac{(c+1-1) m}{m}}}-\frac{1}{\sqrt{\frac{c c}{m}}} \frac{c}{\sqrt{\frac{m}{c}+\frac{m(m-c)}{m-1}+\frac{(c-1) m}{m}}} \geq 0
$$

Simplifies to:

$$
\begin{gathered}
\frac{1}{\sqrt{\frac{(m-c-1)}{m-1}+\frac{1}{c+1}+\frac{c}{m}}}-\frac{1}{\sqrt{\frac{m-c}{m-1}+\frac{1}{c}+\frac{c-1}{m}}} \geq 0 \\
\frac{m-c}{m-1}+\frac{1}{c}+\frac{c-1}{m} \geq \frac{(m-c-1)}{m-1}+\frac{1}{c+1}+\frac{c}{m} \Leftrightarrow \frac{1}{m-1}-\frac{1}{m}+\frac{1}{c}-\frac{1}{c+1} \geq 0
\end{gathered}
$$

This holds for $m \geq 2, c \geq 1, c<m$. Regarding (a) the following needs to hold:

$$
\begin{aligned}
& \frac{1}{\sqrt{\frac{m(m-c-1)}{m-1}+\frac{m}{c+1}+\frac{m}{m} c}}\left(\frac{1}{\sqrt{\frac{(c+1)^{2}}{m}}}+\frac{c}{\sqrt{\frac{m(m-c-1)}{m-1}+\frac{m}{c+1}+\frac{m}{m} c}}+\frac{m-c-1}{\sqrt{\frac{(c+1)(m-1)}{m}+\frac{m-1}{m-1}(m-c-2)}}\right)- \\
& \frac{1}{\sqrt{\frac{c(m-1)}{m}+\frac{m-1}{m-1}(m-c-1)}}\left(\frac{c}{\sqrt{\frac{m(m-c)}{m-1}+\frac{m}{c}+\frac{m}{m}(c-1)}}+\frac{m-c-1}{\sqrt{\frac{c(m-1)}{m}+\frac{m-1}{m-1}(m-c-1)}}\right)>0
\end{aligned}
$$

In order to show that (16) holds, it is separated in two parts. The first part (first line of (16)) will be shown to always be bigger than or equal to 1 (see (17) below) while the second part (second line of (16)) is always strictly smaller than 1 (see (24) below).

$$
\begin{aligned}
& \frac{1}{\sqrt{\frac{m(m-c-1)}{m-1}+\frac{m}{c+1}+c}}\left(\frac{1}{\sqrt{\frac{(c+1)(c+1)}{m}}}+\frac{c}{\sqrt{\frac{m(m-c-1)}{m-1}+\frac{m}{c+1}+c}}+\frac{m-c-1}{\sqrt{m-c-2+\frac{(m-1)(c+1)}{m}}}\right) \geq 1 \\
& \frac{\sqrt{m}}{(c+1) \sqrt{\frac{m(m-c-1)}{m-1}+\frac{m}{c+1}+c}}+\frac{c}{\frac{m(m-c-1)}{m-1}+\frac{m}{c+1}+c}+\frac{m-c-1}{\sqrt{\left(m-c-2+\frac{(m-1)(c+1)}{m}\right)\left(\frac{m(m-c-1)}{m-1}+\frac{m}{c+1}+c\right)}} \geq 1 \\
& \sqrt{m} \sqrt{\frac{m(m-c-1)}{m-1}+\frac{m}{c+1}+c} \sqrt{m-c-2+\frac{(m-1)(c+1)}{m}}+ \\
& c(c+1) \sqrt{m-c-2+\frac{(m-1)(c+1)}{m}}+(m-c-1)(c+1) \sqrt{\frac{m(m-c-1)}{m-1}+\frac{m}{c+1}+c} \geq \\
& (c+1)\left(\frac{m(m-c-1)}{m-1}+\frac{m}{c+1}+c\right) \sqrt{m-c-2+\frac{(m-1)(c+1)}{m}}
\end{aligned}
$$


For the conditions for the terms under the square root, see (22) and (23).

$$
\begin{aligned}
& \sqrt{m} \sqrt{\frac{m^{2}(c+2)-m(c+2)-c^{2}-c}{(m-1)(c+1)}} \sqrt{\frac{m^{2}-m-c-1}{m}}+ \\
& c(c+1) \sqrt{\frac{m^{2}-m-c-1}{m}}+(m-c-1)(c+1) \sqrt{\frac{m^{2}(c+2)-m(c+2)-c^{2}-c}{(m-1)(c+1)}} \geq \\
& (c+1)\left(\frac{m^{2}(c+2)-m(c+2)-c^{2}-c}{(m-1)(c+1)}\right) \sqrt{\frac{m^{2}-m-c-1}{m}}
\end{aligned}
$$

Group the first and third term together and the second and last term together.

$$
\begin{aligned}
\sqrt{\frac{m^{2}(c+2)-m(c+2)-c^{2}-c}{(m-1)(c+1)}}\left(\sqrt{m^{2}-m-c-1}+(m-c-1)(c+1)\right) \geq \\
(c+1) \sqrt{\frac{m^{2}-m-c-1}{m}}\left(\frac{m^{2}(c+2)-m(c+2)-c^{2}-c}{(m-1)(c+1)}-c\right)
\end{aligned}
$$

Simplify the right-hand side.

$$
\begin{gathered}
\sqrt{\frac{m^{2}(c+2)-m(c+2)-c^{2}-c}{(m-1)(c+1)}}\left(\sqrt{m^{2}-m-c-1}+(m-c-1)(c+1)\right) \geq \\
\sqrt{m\left(m^{2}-m-c-1\right)}\left(\frac{m(c+2)-\left(c^{2}+2 c+2\right)}{m-1}\right)
\end{gathered}
$$

Remove the denominators.

$$
\begin{array}{r}
\sqrt{(m-1)\left(m^{2}(c+2)-m(c+2)-c^{2}-c\right)}\left(\sqrt{m^{2}-m-c-1}+(c+1)(m-c-1)\right) \\
\geq \sqrt{m(c+1)\left(m^{2}-m-c-1\right)}\left(m(c+2)-\left(c^{2}+2 c+2\right)\right)
\end{array}
$$

(18) holds if both parts (under the square root and what is in the brackets) of the left-hand side of the inequality are bigger than the corresponding parts of the right-hand side. This is checked separately - first the square root parts (see (19) below) of both sides and then the rest (see (21)).

$$
\begin{gathered}
\sqrt{(m-1)\left(m^{2}(c+2)-m(c+2)-c^{2}-c\right)} \geq \sqrt{m(c+1)\left(m^{2}-m-c-1\right)} \\
m^{3}(c+2)-m^{2}(c+2)-m\left(c^{2}+c\right)-m^{2}(c+2)+m(c+2)+c^{2}+c \geq m^{3}(c+1)-m^{2}(c+1)-m c(c+1)-m(c+1) \\
m^{3}-m^{2}(2 c+4-c-1)-m\left(c^{2}+c-c-2-c^{2}-c-c-1\right)+c^{2}+c \geq 0 \\
m^{3}(c+1)-m^{2}(c+3)+m(2 c+3)+c^{2}+c \geq 0
\end{gathered}
$$

(20) is true if $m^{3}(c+1)-m^{2}(c+3) \geq 0$ which is equivalent to $m \geq \frac{c+3}{c+1} \cdot \frac{c+3}{c+1}$ decreases as $c$ increases, its highest value for $c \geq 1$ being 2 and hence $m \geq 2$ always. 
Now the second part of (18):

$$
\begin{gathered}
\sqrt{m^{2}-m-c-1}+(c+1)(m-c-1) \geq m(c+2)-\left(c^{2}+2 c+2\right) \\
\sqrt{m^{2}-m-c-1}+m(c+1)-(c+1)^{2} \geq m(c+2)-(c+1)^{2}-1 \\
\sqrt{m^{2}-m-c-1} \geq m-1 \Leftrightarrow m^{2}-m-c-1 \geq m^{2}-2 m+1 \Leftrightarrow m \geq c+2
\end{gathered}
$$

Therefore (19) and (21) hold for $m \geq c-2$ and so does (18). What is left is to check is if (18) also holds for $m=c+1$. Substituting $c=m-1$ in (18) yields:

$$
\begin{gathered}
\sqrt{(m-1)\left(m^{2}(m+1)-m(m+1)-m(m-1)\right)}\left(\sqrt{m^{2}-m-m}+m(m-m)\right) \\
\geq \sqrt{m^{2}\left(m^{2}-m-m\right)}(m(m+1)-((m-1)(m+1)+2)) \\
\sqrt{m^{2}(m-1)^{2}} \sqrt{m^{2}-2 m} \geq \sqrt{m^{3}(m-2)}(m-1)
\end{gathered}
$$

This holds with equality. So, (18) and hence (17) holds also for $m=c+1$. Therefore, (17) is true for $m \geq c+1$. Here,

$$
\begin{gathered}
m-c-2+\frac{(m-1)(c+1)}{m}>0 \\
\left(\frac{m}{m-1}\right)(m-c-1)+\frac{m}{c+1}+c>0
\end{gathered}
$$

need to hold. (23) always holds for $m \geq c+1, c \geq 1$ and $m \geq 2$. Now consider (22).

$$
m^{2}-m c-2 m+m c-c+m-1 \geq 0 \Leftrightarrow m^{2}-m-c-1 \geq 0
$$

which holds if $m \geq 2, c \geq 1$ when $m \geq \frac{1+\sqrt{4 c+5}}{2}$ which is always true if $m \geq c+1$. Therefore (17) is true for $m \geq c+1, c \geq 1$ and $m \geq 2$.

Consider the second part of (16):

$$
\begin{gathered}
\frac{1}{\sqrt{\frac{m-1}{m-1}(m-c-1)+\frac{c(m-1)}{m}}}\left(\frac{c}{\sqrt{\frac{m(m-c)}{m-1}+\frac{m}{m}(c-1)+\frac{m}{c}}}+\frac{m-c-1}{\sqrt{\frac{m-1}{m-1}(m-c-1)+\frac{(m-1) c}{m}}}\right)<1 \\
\frac{m-c-1}{m-1-\frac{c}{m}}+\frac{c}{\sqrt{\left(\frac{m(m-c)}{m-1}+c-1+\frac{m}{c}\right)\left(m-1-\frac{c}{m}\right)}}<1 \\
\frac{c}{\frac{m-c-1-m+1}{m-1-\frac{c}{m}}}+\frac{c}{\sqrt{\left(\frac{m(m-c)}{m-1}+c-1+\frac{m}{c}\right)\left(m-1-\frac{c}{m}\right)}}<0 \\
\frac{c}{m-1-\frac{c}{m}}+\frac{c}{\sqrt{\left(\frac{m(m-c)}{m-1}+c-1+\frac{m}{c}\right)\left(m-1-\frac{c}{m}\right)}}<0
\end{gathered}
$$

Simplify.

$$
\frac{\frac{1}{m}-1}{\sqrt{m-1-\frac{c}{m}}}+\frac{1}{\sqrt{\frac{m(m-c)}{m-1}+c-1+\frac{m}{c}}}<0 \Leftrightarrow \frac{\frac{1-m}{m}}{\sqrt{\frac{m^{2}-m-c}{m}}}+\frac{1}{\sqrt{\frac{m(m-c)}{m-1}+c-1+\frac{m}{c}}}<0
$$




$$
\begin{gathered}
\frac{1}{\sqrt{\frac{m^{2}(c+1)-m(c+1)-c^{2}+c}{(m-1) c}}}<\frac{\frac{m-1}{m}}{\sqrt{\frac{m^{2}-m-c}{m}}} \Leftrightarrow \frac{\sqrt{(m-1) c}}{\sqrt{m^{2}(c+1)-m(c+1)-c^{2}+c}}<\frac{\frac{m-1}{m} \sqrt{m}}{\sqrt{m^{2}-m-c}} \\
\frac{\sqrt{c}}{\sqrt{m^{2}(c+1)-m(c+1)-c^{2}+c}}<\frac{\sqrt{m-1}}{\sqrt{m\left(m^{2}-m-c\right)}}
\end{gathered}
$$

This is equivalent to:

$$
\sqrt{\frac{c m\left(m^{2}-m-c\right)}{(m-1)\left(m^{2}(c+1)-m(c+1)-c^{2}+c\right)}}<1
$$

which holds if the numerator is smaller than the denominator, or:

$$
\begin{gathered}
c m\left(m^{2}-m-c\right)<(m-1)\left(m^{2}(c+1)-m(c+1)-c^{2}+c\right) \\
c m^{3}-c m^{2}-c^{2} m<m^{3}(c+1)-m^{2}(c+1)-m c^{2}+m c-m^{2}(c+1)+m(c+1)+c^{2}-c \\
0<m^{3}-m^{2}(c+2)+m(2 c+1)+c^{2}-c
\end{gathered}
$$

This always holds true for $m \geq c+2$. What is left is to check for $m=c+1, m \geq 2$. Then the expression becomes:

$$
\begin{gathered}
m^{3}-m^{2}(m+1)+m(2 m-2+1)+(m-1)(m-2)>0 \\
m^{3}-m^{3}-m^{2}+2 m^{2}-m+m^{2}-m-2 m+2>0 \Leftrightarrow(m-1)^{2}>0
\end{gathered}
$$

This is correct for $m \geq 2$. Therefore (24) is always $<1$, while (17) is always $\geq 1$. Therefore (16) is always true. Hence steps (iii) and (iv) are always possible. 


\section{B.2 Supplementary Materrials - Extensions}

One can view the heuristic investigated in this paper and the equal split of resources between different neighbours as extreme cases of a common model, which just varies the weight that a node puts of the degree of its neighbours. In the case of equal split the degree of a neighbour plays no role, while for the heuristic used in this paper it is inversely proportional to the resources allocation. A more general version of the payoff could be expressed in the following way:

$$
u_{i}=\sum_{n \in N_{i}} \sqrt{\frac{\frac{1}{d_{n}^{\alpha}}}{\sum_{j \in N_{i}} \frac{1}{d_{j}^{\alpha}}} \frac{\frac{1}{d_{i}^{\alpha}}}{\sum_{l \in N_{n}} \frac{1}{d_{l}^{\alpha}}}}=\frac{1}{\sqrt{\sum_{j \in N_{i}}\left(\frac{d_{i}}{d_{j}}\right)^{\alpha}}}\left(\sum_{n \in N_{i}} \frac{1}{\sqrt{\sum_{l \in N_{n}}\left(\frac{d_{n}}{d_{l}}\right)^{\alpha}}}\right)
$$

where $\alpha=0$ corresponds to the case in which the resources are spread equally amongst all neighbours and $\alpha=1$ is the current heuristic. For $0 \leq \alpha \leq 1$ Propositions 3 and 5 hold.

Proof of Proposition 3EXT: In a fully connected graph every node has payoff 1 . In order for the complete graph to be stable, removing a link should be equally good or worse than the status quo for both nodes that are connected so that they decide not to disconnect. For a complete graph with $n$ nodes, given that $1 \geq \alpha \geq 0$ this is equivalent to:

$$
\begin{gathered}
\frac{1}{\sqrt{(n-2) \frac{(n-2)^{\alpha}}{(n-1)^{\alpha}}}}\left(\frac{n-2}{\sqrt{2 \frac{(n-1)^{\alpha}}{(n-2)^{\alpha}}+\frac{(n-1)^{\alpha}}{(n-1) \alpha}(n-3)}}\right) \leq 1 \Leftrightarrow \frac{n-2}{\sqrt{\frac{(n-2)\left(2(n-1)^{\alpha}+(n-3)(n-2)^{\alpha}\right)}{(n-1)^{\alpha}}}} \leq 1 \\
(n-2)(n-1)^{\alpha} \leq 2(n-1)^{\alpha}+(n-3)(n-2)^{\alpha} \Leftrightarrow(n-4)(n-1)^{\alpha} \leq(n-3)(n-2)^{\alpha} \\
\left(\frac{n-1}{n-2}\right)^{\alpha} \leq \frac{n-3}{n-4}
\end{gathered}
$$

Both parts of the inequality are bigger than 1 for all positive $n>4$. Moreover, as $\alpha$ comes closer to 1 , the left-hand side grows. Therefore, the biggest value for the left-hand side is $\frac{n-1}{n-2}$. In this case the inequality still holds for $n>4$. What is left is to check if this holds for $n \in\{3,4\}$. For $n=3$ :

$$
\frac{1}{\sqrt{1 \frac{1^{\alpha}}{2^{\alpha}}}} \frac{1}{\sqrt{2 \frac{2^{\alpha}}{1^{\alpha}}}} \leq 1
$$

For $n=4$ :

$$
\frac{1}{\sqrt{2 \frac{2^{\alpha}}{3^{\alpha}}}} \frac{2}{\sqrt{2 \frac{3^{\alpha}}{2^{\alpha}}+1}} \leq 1
$$

Both conditions hold. Therefore, no two nodes would decide to disconnect in a complete graph. 
Proof of Proposition 5EXT: To prove the statement it is sufficient to show that in any regular network with degrees $n$ and $1 \geq \alpha \geq 0$ which is not complete two of its nodes would want to form a connection. This is equivalent to the condition:

$\frac{1}{\sqrt{\frac{(n+1)^{\alpha}}{(n+1)^{\alpha}}+n \frac{(n+1)^{\alpha}}{n^{\alpha}}}}\left(\frac{1}{\sqrt{\frac{(n+1)^{\alpha}}{(n+1)^{\alpha}}+n \frac{(n+1)^{\alpha}}{n^{\alpha}}}}+\frac{\beta}{\sqrt{2 \frac{n^{\alpha}}{(n+1)^{\alpha}}+(n-2) \frac{n^{\alpha}}{n^{\alpha}}}}+\frac{n-\beta}{\sqrt{\frac{n^{\alpha}}{(n+1)^{\alpha}}+(n-1) \frac{n^{\alpha}}{n^{\alpha}}}}\right)>1$

where $\beta \geq 0$ is the number of agents that are mutual neighbours of the two connecting nodes.

The condition can be simplified.

$\frac{\sqrt{n^{\alpha}}}{\sqrt{n^{\alpha}+n(n+1)^{\alpha}}}\left(\frac{\sqrt{n^{\alpha}}}{\sqrt{n^{\alpha}+n(n+1)^{\alpha}}}+\frac{\beta \sqrt{(n+1)^{\alpha}}}{\sqrt{2 n^{\alpha}+(n-2)(n+1)^{\alpha}}}+\frac{(n-\beta) \sqrt{(n+1)^{\alpha}}}{\sqrt{n^{\alpha}+(n-1)(n+1)^{\alpha}}}\right)>1$

Since

$$
\frac{\sqrt{(n+1)^{\alpha}}}{\sqrt{2 n^{\alpha}+(n-2)(n+1)^{\alpha}}} \geq \frac{\sqrt{(n+1)^{\alpha}}}{\sqrt{n^{\alpha}+(n-1)(n+1)^{\alpha}}}
$$

holds for every $n \geq 1,1 \geq \alpha \geq 0$, taking $\beta=0$ presents the worst-case scenario for Inequality $(25)$, i.e. the case in which its left-hand side has the lowest possible value, presenting the lowest incentive for the two nodes to connect. This leaves:

$$
\begin{gathered}
\frac{\sqrt{n^{\alpha}}}{\sqrt{n^{\alpha}+n(n+1)^{\alpha}}}\left(\frac{\sqrt{n^{\alpha}}}{\sqrt{n^{\alpha}+n(n+1)^{\alpha}}}+\frac{n \sqrt{(n+1)^{\alpha}}}{\sqrt{n^{\alpha}+(n-1)(n+1)^{\alpha}}}\right)>1 \\
\frac{n \sqrt{n^{\alpha}(n+1)^{\alpha}}}{n^{\alpha}+n(n+1)^{\alpha}}+\frac{n \sqrt{n^{\alpha}(n+1)^{\alpha}}}{\sqrt{\left(n^{\alpha}+(n-1)(n+1)^{\alpha}\right)\left(n^{\alpha}+n(n+1)^{\alpha}\right)}}>1 \\
\frac{n(n+1)^{\alpha}}{\sqrt{\left(n^{\alpha}+(n-1)(n+1)^{\alpha}\right)\left(n^{\alpha}+n(n+1)^{\alpha}\right)}}>\frac{n+n(n+1)^{\alpha}}{n^{\alpha}+n} \\
\frac{\sqrt{n^{\alpha}}}{\sqrt{n^{\alpha}+(n-1)(n+1)^{\alpha}}}>\frac{\sqrt{n^{\alpha}+n(n+1)^{\alpha}}}{n^{2 \alpha}+n^{\alpha+1}(n+1)^{\alpha}>n^{\alpha}(n+1)^{\alpha}+(n-1)(n+1)^{2 \alpha}} \\
\left(\frac{n}{n+1}\right)^{2 \alpha}+\left(\frac{n}{n+1}\right)^{\alpha}(n-1)-(n-1)>0
\end{gathered}
$$

This expression is at its lowest for $\alpha$ as big as possible. Taking $\alpha=1$, the expression is always true for $n \geq 3$.

Proof of Proposition 6EXT: This proof looks at the first two parts of Proposition 6.

A) Line: The two ends would want to connect, since:

$$
\frac{1}{\sqrt{\frac{1^{\alpha}}{2^{\alpha}}}} \frac{1}{\sqrt{\frac{2^{\alpha}}{1^{\alpha}}+\frac{2^{\alpha}}{1^{\alpha}}}}<1 ; \frac{1}{\sqrt{\frac{1^{\alpha}}{2^{\alpha}}}} \frac{1}{\sqrt{\frac{2^{\alpha}}{1^{\alpha}}+\frac{2^{\alpha}}{2^{\alpha}}}}<1
$$


The first inequality refers to a line of length 3 , while the second one covers all other cases.

B) Star: It is sufficient to show that two of the periphery nodes of a star network with $n \geq 3$ would want to form a link. The condition is:

$$
\frac{1}{\sqrt{\frac{2^{\alpha}}{(n-1)^{\alpha}}+\frac{2^{\alpha}}{2^{\alpha}}}}\left(\frac{1}{\sqrt{\frac{(n-1)^{\alpha}}{2^{\alpha}} 2+\frac{(n-1)^{\alpha}}{1^{\alpha}}(n-3)}}+\frac{1}{\sqrt{\frac{2^{\alpha}}{(n-1)^{\alpha}}+\frac{2^{\alpha}}{2^{\alpha}}}}\right) \geq \frac{1}{\sqrt{\frac{1^{\alpha}}{(n-1)^{\alpha}}}} \frac{1}{\sqrt{(n-1) \frac{(n-1)^{\alpha}}{1^{\alpha}}}}
$$

Simplified:

$$
\begin{gathered}
\frac{1}{\sqrt{\frac{2^{\alpha}+(n-1)^{\alpha}}{(n-1)^{\alpha}}}}\left(\frac{1}{\sqrt{(n-1)^{\alpha}\left(\frac{2+2^{\alpha}(n-3)}{2^{\alpha}}\right)}}+\frac{1}{\sqrt{\frac{2^{\alpha}+(n-1)^{\alpha}}{(n-1)^{\alpha}}}}\right) \geq \frac{1}{\sqrt{n-1}} \\
\frac{2^{\alpha}}{\sqrt{\left(2^{\alpha}+(n-1)^{\alpha}\right)\left(2+2^{\alpha}(n-3)\right)}}+\frac{(n-1)^{\alpha}}{2^{\alpha}+(n-1)^{\alpha}} \geq \frac{1}{\sqrt{n-1}}
\end{gathered}
$$

Taking the second part:

$$
\frac{(n-1)^{\alpha}}{2^{\alpha}+(n-1)^{\alpha}} \geq \frac{1}{\sqrt{n-1}}
$$

$\frac{(n-1)^{\alpha}}{2^{\alpha}+(n-1)^{\alpha}}$ is bigger than $1 / 2$ for $n=4$ and it has a positive first derivative w.r.t. $n$ so as $n$ increases the term becomes bigger. The derivative is:

$$
\frac{\alpha(n-1)^{\alpha}}{2^{\alpha}+(n-1)^{\alpha}}-\frac{\alpha(n-1)^{2 \alpha}}{\left(2^{\alpha}+(n-1)^{\alpha}\right)^{2}}
$$

The first term of (26) is always positive. Finally, a specific check for $n=3$ shows that (26) holds for integers $n \geq 3$. 


\section{B.3 Supplementary Matreials - Cycle with Fixed Order}

This is a description of a short cycle that can occur within this setup if a specific order of operations is followed. There are two procedures - addition and deletion of a link. The addition checks if one link could be added. The deletion checks if any number of links could be gradually removed. First is the addition procedure after which the deletion procedure begins. The deletion cuts links step by step (restarting the checking procedure after every change) until no links can be removed and only then can the addition procedure start again. The deletion and addition procedures alternate until no links can be added or removed and then the process stops.

Importantly, there is a fixed order followed for every check after the adjacency matrix has been changed (this includes every time a link has been severed) - always starting from the cell $(1,1)$, continuing along the row and going to the next row after checking the whole row. Since the adjacency matrix is symmetric, only the values above the main diagonal are checked.

Consider Figure 3 (a). The overall welfare is $W=6.813$. The contributions of each node to each node and the payoffs at each node are: (i) for 1 : $\left(\frac{1}{5}, \frac{1}{5}, \frac{3}{10}, \frac{3}{10}\right)$, payoff 1.322 ; (ii) for 2 : $\left(\frac{1}{6}, \frac{1}{6}, \frac{1}{6}, \frac{1}{4}, \frac{1}{4}\right)$, payoff 1.275 ; (iii) for $3: \frac{3}{13}, \frac{5}{13}, \frac{5}{13}$, payoff 1.005 ; (iv) for $4,5: \frac{15}{47}, \frac{12}{47}, \frac{20}{47}$, payoff 0.863 ; (v) for $6,7: \frac{5}{9}, \frac{4}{9}$, payoff 0.742 .

In Figure 3 (b) overall welfare is $W=6.752$ and the difference to (a) is that nodes 1 and 3 are connected. To see why consider the corresponding payoffs: (i) for 1,2 : $\frac{1}{\sqrt{\frac{52}{3}+\frac{5}{4}+\frac{52}{2}}}$ $\left(\frac{2}{\sqrt{\frac{3}{4}+\frac{23}{5}}}+\frac{1}{\sqrt{\frac{42}{3}+\frac{24}{5}}}+\frac{2}{\sqrt{\frac{22}{5}}}\right)=1.341$; (ii) for $3: \frac{1}{\sqrt{\frac{42}{3}+\frac{42}{5}}}\left(\frac{2}{\sqrt{\frac{3}{4}+\frac{23}{5}}}+\frac{2}{\sqrt{\frac{5}{4}+\frac{25}{3}+\frac{52}{2}}}\right)=1.006$; (iii) for 4,5 : $\frac{1}{\sqrt{\frac{3}{4}+\frac{32}{5}}}\left(\frac{1}{\sqrt{\frac{42}{3}+\frac{24}{5}}}+\frac{2}{\sqrt{\frac{5}{4}+\frac{25}{3}+\frac{52}{2}}}\right)=0.809$; (iv) for 6,7 : $\frac{1}{\sqrt{\frac{22}{5}}} \frac{2}{\sqrt{\frac{5}{4}+\frac{25}{3}+\frac{52}{2}}}=$ 0.722 .

A connection between 1 and 2 would make 1 worse off (cf. Figure $3(\mathrm{~d})$ ), since the payoff of 1 would be $\frac{1}{\sqrt{\frac{52}{2}+\frac{5}{6}+\frac{52}{3}}}\left(\frac{2}{\sqrt{\frac{2}{5}+\frac{2}{6}}}+\frac{2}{\sqrt{\frac{3}{3}+\frac{3}{5}+\frac{3}{6}}}+\frac{1}{\sqrt{\frac{62}{2}+\frac{6}{5}+\frac{63}{3}}}\right)=1.318$. And as a result nodes 1 and 3 connect, since both are better off connected. However, now no two nodes should be willing to sever their link. Clearly, 1 and 3 would not want to disconnect.

If 1 and 4 disconnect their corresponding payoffs would be: (i) for 1 : $\frac{1}{\sqrt{\frac{4}{4}+\frac{4}{3}+\frac{42}{2}}}\left(\frac{1}{\sqrt{\frac{3}{5}+\frac{23}{4}}}+\right.$ $\left.\frac{1}{\sqrt{\frac{4}{2}+\frac{4}{4}+\frac{4}{3}+\frac{4}{5}}}+\frac{2}{\sqrt{\frac{2}{5}+\frac{2}{4}}}\right)=1.287$; (ii) for $4: \frac{1}{\sqrt{\frac{2}{4}+\frac{2}{5}}}\left(\frac{1}{\sqrt{\frac{4}{2}+\frac{4}{4}+\frac{4}{3}+\frac{4}{5}}}+\frac{1}{\sqrt{\frac{5}{4}+\frac{5}{3}+\frac{53}{2}}}\right)=0.792$. So, they would not disconnect. Same reasoning holds for 1 and 5 .

If 1 and 6 disconnect their corresponding payoffs would be: (i) for $1: \frac{1}{\sqrt{\frac{42}{3}+\frac{4}{4}+\frac{4}{2}}}\left(\frac{1}{\sqrt{\frac{2}{4}+\frac{2}{5}}}+\right.$ $\left.\frac{1}{\sqrt{\frac{4}{4}+\frac{42}{3}+\frac{4}{5}}}+\frac{2}{\sqrt{\frac{3}{5}+\frac{23}{4}}}\right)=1.221$; (ii) for 6 : $\frac{1}{\sqrt{\frac{1}{5}} \sqrt{\frac{5}{4}+\frac{25}{3}+\frac{5}{2}+5 \frac{1}{1}}}=0.643$ So, they would not disconnect. Same reasoning holds for 1 and 7 and 2 and all its neighbours. If 3 and 4 
disconnect their corresponding payoffs would be: (i) for $3: \frac{1}{\sqrt{\frac{3}{3}+\frac{32}{5}}}\left(\frac{1}{\sqrt{\frac{3}{3}+\frac{23}{5}}}+\frac{2}{\sqrt{\frac{35}{2}+\frac{25}{2}}}\right)=$ 0.836; (ii) for 4: $\frac{2}{\sqrt{\frac{42}{5}} \sqrt{\frac{35}{2}+\frac{25}{3}}}=0.480$. So, they would not disconnect. Same reasoning holds for 3 and 5. Therefore, no nodes would want to disconnect in Figure 3 (b). This leads to the next check if additional nodes want to form a connection.

In Figure 3 (c) the overall welfare is $W=6.719$ and nodes 1 and 2 have a link. To see why, consider the corresponding payoffs of 1,2 which are $\frac{1}{\sqrt{\frac{62}{2}+\frac{6}{6}+\frac{6}{4}+\frac{62}{3}}}\left(\frac{1}{\sqrt{\frac{42}{6}+\frac{42}{3}}}+\right.$ $\left.\frac{1}{\sqrt{\frac{62}{2}+\frac{6}{6}+\frac{6}{4}+\frac{62}{3}}}+\frac{2}{\sqrt{\frac{3}{4}+\frac{32}{6}}}+\frac{2}{\sqrt{\frac{22}{6}}}\right)=1.342$. So, they would connect since it improves both of them strictly.

At this point, 3 would choose to disconnect from 1 (Figure $3(\mathrm{~d})$ ) since it gets a payoff of $\frac{1}{\sqrt{\frac{23}{3}+\frac{3}{5}}}\left(\frac{2}{\sqrt{\frac{3}{3}+\frac{3}{6}+\frac{3}{5}}}+\frac{1}{\sqrt{\frac{36}{6}+\frac{6}{5}+\frac{6}{2}}}\right)=1.087$ as compared to the payoff in (c), $\frac{1}{\sqrt{\frac{24}{3}+\frac{42}{6}}}\left(\frac{2}{\sqrt{\frac{32}{6}+\frac{3}{4}}}+\right.$ $\left.\frac{2}{\sqrt{\frac{62}{2}+\frac{6}{6}+\frac{6}{4}+\frac{62}{3}}}\right)=1.039$. There the overall welfare is $W=6.776$.

In Figure 3 (e), 2 would choose to disconnect from 1 since it gets a payoff of 1.341 (same as in (a)) as compared to the payoff in (d), $\frac{1}{\sqrt{\frac{52}{2}+\frac{5}{6}+\frac{52}{3}}}\left(\frac{2}{\sqrt{\frac{2}{5}+\frac{2}{6}}}+\frac{2}{\sqrt{\frac{3}{3}+\frac{3}{5}+\frac{3}{6}}}+\frac{1}{\sqrt{\frac{62}{2}+\frac{6}{5}+\frac{63}{3}}}\right)=$ 1.318. This completes the cycle. It must be noted that the overall welfare in the different instances first decreases and then increases. 


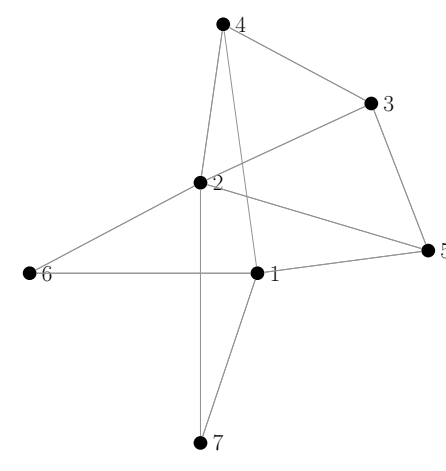

(a) Initial stage

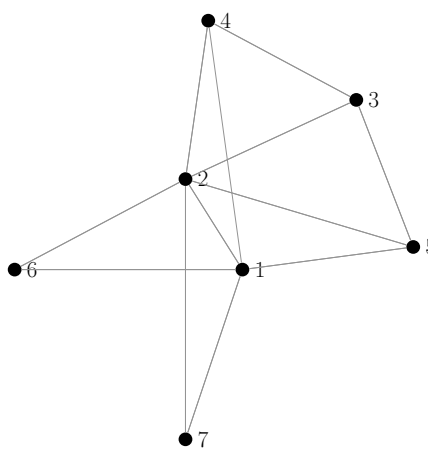

(d) 1 and 3 disconnect

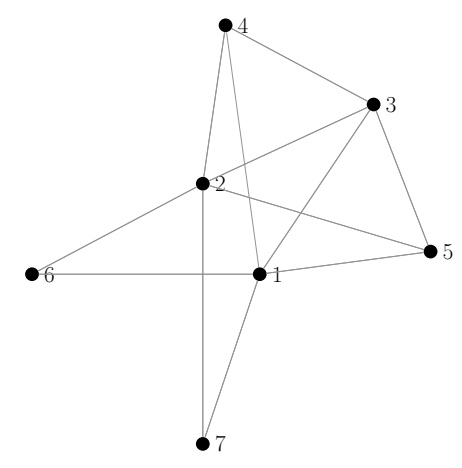

(b) 1 and 3 connect

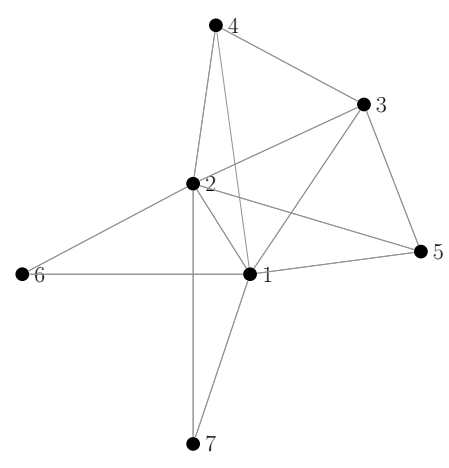

(c) 1 and 2 connect

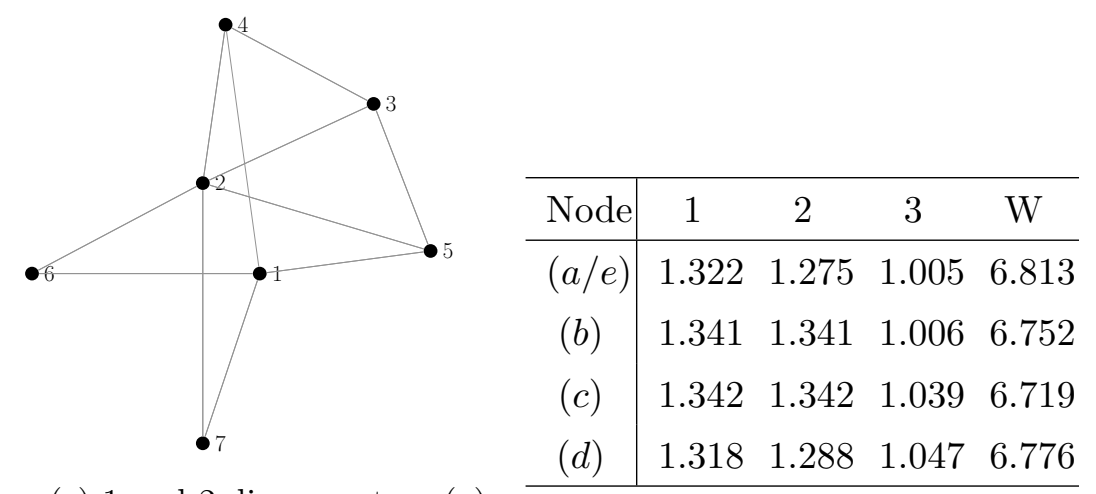

(f) Node payoffs and welfare (W)

Figure 3: Cycle with fixed order for $n=7$ 